\title{
EL INVENTARIO FONÉMICO NASAL DEL ESPAÑOL: UN ESTUDIO COMPARATIVO
}

\author{
Carlos-Eduardo Piñeros \\ Universidad de Auckland
}

\section{RESUMEN}

Este trabajo investiga la composición del inventario fonémico nasal del español usando la UPSID (UCLA Phonological Segment Inventory Database) como marco de comparación. Se encontró que el inventario /m, n, n/ concuerda con una tendencia translingüística predominante -la elección de /y/ para completar la terna nasal-. El análisis propuesto desde la teoría de optimidad se basa en el supuesto de que, además de una dimensión de MARCACIÓN articulatoria, existe también una dimensión de MARCACIÓN perceptiva, formalizadas ambas en términos de restricciones negativas y sujetas a un orden jerárquico universal. El número de consonantes nasales y los rasgos de lugar que estas puedan tener dependen del rango que cada gramática le asigne a FIEL (lugar nasal) -el principio de fidelidad relevante-con respecto a los integrantes de las dos jerarquías universales de MARCACIÓN. Según este enfoque, la dominación absoluta de Fiel (lugar nasal) por parte de ambas jerarquías de marcación acarrea la ausencia de consonantes nasales, mientras que la promoción gradual de esa restricción de fidelidad conduce a inventarios nasales gradualmente más poblados. Sin embargo, por alto que sea el rango de Fiel (lugar nasal), el orden fijo de las jerarquías de marcación descarta la posibilidad de generar inventarios nasales constituidos exclusivamente por unidades marcadas (p. ej. la terna /n, n, $\mathrm{y} /$ ). Solo en los inventarios que incluyen las unidades menos marcadas pueden surgir también las unidades más marcadas.

Palabras clave: nasales, marcación, rasgos de lugar, coronal, labial, rango universal.

\section{ABSTRACT}

This paper investigates the composition of the Spanish phonemic nasal inventory using the UPSID (UCLA Phonological Segment Inventory Database) as a framework of comparison. It was found that the inventory $/ \mathrm{m}, \mathrm{n}, \mathrm{n} / \mathrm{agrees}$ with a predominant crosslinguistic tendency -the inclusion of $/ \mathrm{m} /$ and $/ \mathrm{n} /-$ but disagrees with another predominant crosslinguistic tendency-the selection of $/ \mathrm{y} /$ to complete the nasal triad-. The Optimality-theoretic analysis proposed herein is based on the assumption that, in addition to an articulation markedness dimension, there is also a perception markedness dimension, both of which are formalized in terms of negative constraints and assumed to be subject to a universal hierarchical order. The number of nasal consonants and the place features that they may have depend on the rank that individual grammars assign to FAITH (nasal place) -the pertinent faithfulness principle- with respect to the members of two universal markedness hierarchies. 
According to this approach, absolute domination of FAITH (nasal place) by both markedness hierarchies leads to the absence of nasal consonants, while the gradual promotion of this faithfulness constraint allows for nasal inventories that are gradually more populated. Yet, regardless of how highly FAITH (nasal place) is promoted, the fixed internal order of the markedness hierarchies rules out the possibility of generating nasal inventories comprising exclusively marked units (e. g. the triad $/ \mathrm{n}, \mathrm{n}, \mathrm{y} /$ ). Only in those inventories were the least marked units are present can the most marked units arise as well.

Key Words: nasals, markedness, place features, coronal, labial, universal ranking.

RECIBIDO: 04/12/2009

APROBADO: 01/06/2010

\section{INTRODUCCIÓN: SimilitUd CON OTROS INVENTARIOS FONÉMICOS NASALES}

Es bien sabido que el inventario fonémico del español incluye varias consonantes oclusivas nasales, las cuales contrastan en la dimensión del lugar de articulación. Se distingue la bilabial $/ \mathrm{m} /$, la alveolar $/ \mathrm{n} / \mathrm{y}$ la palatal $/ \mathrm{n} /$. Tal inventario nasal concuerda en gran medida con los inventarios nasales utilizados por otras lenguas que también distinguen varias consonantes nasales. El inglés, por ejemplo, posee tantas oclusivas nasales como el español y concuerda adicionalmente en incluir una bilabial y una alveolar. Esto se puede constatar en (1), donde vemos que, si no fuese porque el inglés opta por completar su terna con la velar $/ \mathrm{y} /$, los inventarios nasales de las dos lenguas serían idénticos ${ }^{1}$.

\begin{tabular}{|c|c|c|c|c|}
\hline & Bilabial & Alveolar & Palatal & Velar \\
\hline \multirow{2}{*}{ a. Español } & $/ \mathrm{m} /$ ato & /n/ato & $/ \mathrm{n} /$ ato & - \\
\hline & $\mathrm{do} / \mathrm{m} / \mathrm{a}$ & $\mathrm{do} / \mathrm{n} / \mathrm{a}$ & $\mathrm{do} / \mathrm{n} / \mathrm{a}$ & - \\
\hline \multirow{2}{*}{ b. Inglés ${ }^{2}$} & $\mathrm{ra} / \mathrm{m} /$ & $\mathrm{ra} / \mathrm{n} /$ & - & $\mathrm{ra} / \mathrm{y} /$ \\
\hline & $\mathrm{su} / \mathrm{m} /$ & $\mathrm{su} / \mathrm{n} /$ & - & $\mathrm{su} / \mathrm{y} /$ \\
\hline
\end{tabular}

TABLA 1: Triple oposición entre consonantes nasales en español e inglés.

\footnotetext{
1 Este artículo incorpora varias sugerencias ofrecidas por dos revisores anónimos a quienes deseo expresar mi agradecimiento por sus valiosos comentarios.

${ }^{2}$ Las glosas correspondientes a los ejemplos del inglés son las siguientes.

$\begin{array}{lll}\text { 'carnero' } & \text { 'corrió' } & \text { 'tocó el timbre' } \\ \text { 'suma' } & \text { 'sol' } & \text { 'cantado/a' }\end{array}$
}


Un aspecto clave de la semejanza existente entre los inventarios nasales del español y del inglés es el empleo de Consonantes Nasales Primarias (CNPs). Según Ferguson 1963, una CNP es un fonema cuyo alófono más característico es una oclusiva nasal sonora simple; esto es, un segmento cuya articulación involucra un solo cierre oral completo, apertura del canal velofaríngeo y vibración de las cuerdas vocales. Una Consonante Nasal Secundaria (CNS) es, en contraste, un fonema nasal cuyo alófono más característico no es una oclusiva nasal sonora simple. Lo que se quiere decir con esto es que es posible que tal fono se articule sin cierre oral completo o con más de una constricción oral, con un tipo inusual de fonación (p. ej. sordez, glotalización, murmullo), o puede ser una obstruyente prenasalizada/posnasalizada o un clic nasalizado. De acuerdo con estas definiciones, los fonemas nasales del español y del inglés deben clasificarse como CNPs en vista de que, excepto cuando existen factores contextuales que los obligan a cambiar, sus alófonos son segmentos que poseen el tipo de cierre oral, el tipo de apertura velofaríngea y el tipo de fonación característicos de las consonantes oclusivas nasales sonoras simples ${ }^{3}$.

Dado que el español es una lengua románica, no es insólito que su inventario nasal sea idéntico al de otras lenguas románicas, como por ejemplo el catalán, que también distingue $/ \mathrm{m} /, / \mathrm{n} / \mathrm{y} / \mathrm{n} /$ (p. ej. ca[m] ella 'camella', ca[n] ella 'caño de una fuente', ca[n] ella 'canela') ${ }^{4}$. De manera similar, la menor, pero aun extensa, semejanza que existe entre los inventarios nasales del español y del inglés no debe ser motivo de extrañeza si tenemos en cuenta que el inglés pertenece al grupo de las lenguas germánicas, relacionadas con las lenguas románicas por provenir del mismo ancestro, el indoeuropeo. Lo que es verdaderamente digno de atención es que, aun en lenguas con las que el español no tiene ninguna relación genética, encontramos un alto grado de semejanza con respecto a los fonemas seleccionados para componer los inventarios nasales. Para demostrar este punto, nos valdremos de la base de datos conocida como UPSID (UCLA Phonological Segment Inventory Database), accesible a través de Internet <http://web.phonetik.uni-frankfurt.de/upsid>.

Las lenguas incluidas en la UPSID han sido escogidas con el propósito de obtener una muestra representativa y genéticamente balanceada

\footnotetext{
${ }^{3}$ Desviaciones con respecto a esta norma ocurren en ambas lenguas, pero están limitadas a contextos específicos o son evoluciones dialectales. Un caso bien conocido es la tendencia de las oclusivas nasales del inglés a ensordecerse cuando van precedidas por una fricativa sorda dentro del ataque de la misma sílaba (p. ej. [sm] all 'pequeño' y [sn] ail ‘caracol'). En el caso del español, una desviación relacionada con la constricción oral ocurre en variedades dialectales en las que las consonantes oclusivas nasales finales de palabra tienden a velarizarse y pueden perder su cierre oral completo (p. ej. so/n/ puede realizarse como so[y] o so $[\tilde{\gamma}]$ ).

4 Agradezco a Rosa-María Pérez Castañeda el haberme facilitado varios ejemplos de tripletes nasales existentes en catalán.
} 
de las lenguas que existen en el mundo. El principio que se ha adoptado para garantizar una cuota apropiada es incluir todas las familias de lenguas que se conocen y no más de una lengua por cada una de las ramas en que estas se dividen (Maddieson 1984, p. 5). En el año en que escribimos (2009), la UPSID cuenta con un total de 451 lenguas, lo cual representa una expansión del $42.27 \%$ con respecto a las 317 lenguas que contenía el año en que Maddieson publicó su estudio. Cabe destacar que, de estas 451 lenguas, solamente $23(5.01 \%)$ pertenecen a la familia indoeuropea.

Escudriñar las lenguas de la UPSID nos ayudará a ubicar el inventario nasal del español dentro de un contexto translingüístico más amplio, pero antes de proceder a ello es necesario hacer algunas aclaraciones con respecto a la notación. Siguiendo a Maddieson 1984, nos valemos del símbolo /*n/ para subsumir dos CNPs coronales: la alveolar /n/ y la dental /n/, cuya clasificación independiente no se logró siempre en la UPSID, debido a que algunas de las fuentes utilizadas no especifican esa información. Otra convención que adoptamos de Maddieson es el símbolo /*n/, el cual permite subsumir dos CNPs dorso-coronales, la

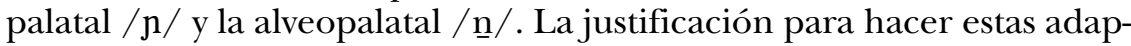
taciones es que, en cada caso, se trata de dos puntos de articulación emparentados, dado que corresponden a constricciones creadas por los mismos órganos articuladores -la corona, en el caso de $/ \mathrm{n} / \mathrm{y} / \mathrm{n}_{\mathrm{n}} /$, y el dorso y la corona, en el caso de $/ \mathrm{n} / \mathrm{y} / \underline{\mathrm{n}} /-$.

Una inspección de la UPSID revela que la mayoría de las lenguas incluyen tanto /m/ como /*n/ en sus inventarios nasales. El fonema /m/ aparece en 425 de las 451 lenguas $(94.24 \%)$, mientras que /*n/ ocurre en 445 lenguas $(98.67 \%)$. Aunque hay un grupo de 17 lenguas que no tienen $\mathrm{ni} / \mathrm{m} / \mathrm{ni} /{ }^{*} \mathrm{n} /(3.77 \%)$ y también hay 11 lenguas que incluyen uno de estos fonemas sin incluir el otro $(2.43 \%)$, es indudable que la gran mayoría los emplea ambos, 423 lenguas o 93.79\%. Otro hecho que señala que $/ \mathrm{m} / \mathrm{y} /{ }^{*} \mathrm{n} /$ son extremadamente abundantes en los inventarios nasales es que, entre las 138 lenguas de la UPSID que emplean solamente dos CNPs, hay $136(98.55 \%)$ que eligen precisamente esos dos fonemas.

Las cifras que acabamos de presentar son prueba de que la semejanza que existe entre los inventarios nasales del español y del inglés no es una mera coincidencia ni tampoco una idiosincrasia de la familia indoeuropea, sino más bien un patrón recurrente y ampliamente difundido. La implicación que esto tiene para el presente estudio es que la participación de $/ \mathrm{m} / \mathrm{y} /{ }^{*} \mathrm{n} /$ en el inventario nasal del español no se puede achacar a normas específicas de la gramática de esta lengua sino que es necesario buscar una explicación basada en principios lingüísticos universales. En consecuencia, una pregunta esencial que tenemos que plantearnos es la siguiente: ¿cuáles son los principios universales 
responsables de que el español, al igual que la mayoría de las lenguas del mundo, incluya $/ \mathrm{m} / \mathrm{y} /{ }^{*} \mathrm{n} /$ en su inventario nasal?

Otra información pertinente que nos suministra la UPSID es que los inventarios nasales compuestos por tres CNPs son bastante frecuentes. De hecho, aunque los inventarios nasales compuestos por dos y cuatro CNPs son también bastante abundantes $(30.60 \%$ y $25.94 \%$, respectivamente), aquellos que constan de tres CNPs son ligeramente más frecuentes. En su tamaño actual, la UPSID contiene 143 lenguas que contrastan tres CNPs $(31.71 \%)^{5}$. Al escudriñar este grupo de lenguas, encontramos los cinco subgrupos presentados en la tabla (2), donde se puede verificar que la terna nasal usada por el español, /m, ${ }^{*} \mathrm{n},{ }^{*} \mathrm{n} /$, no es la más común, sino que constituye un patrón secundario (31.47\%). Es la terna nasal utilizada por el inglés, /m, *n, y/, la que representa el patrón principal $(65.03 \%)$. Al lado de estos dos patrones predominantes, existe un patrón recesivo representado por un puñado de lenguas que crean la terna /m, *n, $n /$ reclutando la retrofleja / $\mathrm{n} /$ como tercer elemento $(2.10 \%)$. El telugu, perteneciente a la familia dravídica, es un ejemplo representativo. Adicionalmente, existen dos lenguas aisladas, isoko y japonés, que completan sus ternas nasales con la dental / $\mathrm{n} / \mathrm{o}$ la uvular $/ \mathrm{N} /(0.70 \%$ cada una $)$.

\begin{tabular}{|c|c|c|c|}
\hline Ternas & Número de lenguas & Porcentaje & Representante \\
\hline a. $/ \mathrm{m}, * \mathrm{n}, \mathrm{n} /$ & 93 & $65.03 \%$ & Inglés \\
\hline b. $/ \mathrm{m}, * \mathrm{n}, * \mathrm{n} /$ & 45 & $31.47 \%$ & Español \\
\hline c. $/ \mathrm{m}, * \mathrm{n}, \mathrm{n} /$ & 3 & $2.10 \%$ & Telugu \\
\hline d. $/ \mathrm{m}, \mathrm{n}, \mathrm{n} /$ & 1 & $0.70 \%$ & Isoko \\
\hline e. $/ \mathrm{m}, \mathrm{n}, \mathrm{N} /$ & 1 & $0.70 \%$ & Japonés \\
\hline TOTALES & 143 & $100.00 \%$ & \\
\hline
\end{tabular}

TABLA 2: Lenguas de la UPSID con inventarios nasales compuestos por tres $\mathrm{CNPs}^{6}$.

5 En 1984, la UPSID contenía 95 lenguas con tres CNPs (Maddieson 1984). Dado que esta cifra equivale a $29.97 \%$, podemos verificar que las 134 lenguas que se han añadido en los últimos veinticinco años no han causado un cambio significativo en el porcentaje de lenguas con tres CNPs.

${ }^{6}$ Maddieson 1984 da cuenta de porcentajes similares. En esa época, la UPSID contenía un total de 95 lenguas con tres CNPs, entre las cuales había 65 (68.42\%) que seguían el patrón ejemplificado por el inglés, $27(28.42 \%)$ que seguían el patrón ejemplificado por el español y $2(2.10 \%)$ que seguían el patrón ejemplificado por el telugu. 
Por no ser recurrente, la inclusión de /n $/ \mathrm{o} / \mathrm{N} /$ en las ternas nasales se puede catalogar como una idiosincrasia de los inventarios nasales del isoko y del japonés, lo cual es razonable atribuir a normas específicas de las gramáticas de esas lenguas. En contraste, por verificarse recurrentemente, la inclusión de $/ \mathrm{n} /, / * \mathrm{n} / \mathrm{o} / \mathrm{n} /$ en las ternas nasales da pie a tendencias translingüísticas, las cuales no pueden tener su origen en las normas de una lengua específica sino que deben provenir de principios de la Gramática Universal. De acuerdo con este razonamiento, vamos a dejar de lado los inventarios en (2e-d) para ir a la búsqueda de los principios lingüísticos universales responsables por los inventarios en $(2 \mathrm{a}-\mathrm{c})$.

La pregunta que hay que formular con respecto a (2b) es la siguiente: ¿qué es lo que hace que las lenguas representadas por el español se alejen de la tendencia predominante a completar la terna nasal con / $\mathrm{n} /$ y opten por incluir $/{ }^{*} \mathrm{n} /$ en su lugar? En el caso de las lenguas representadas por el telugu, (2c), el interrogante que surge es similar. ¿Cuál es la razón de que tales lenguas opten por completar la terna nasal con $/ \mathrm{n} /$ en lugar de /n/? Adicionalmente, hay una cuestión relacionada que vale la pena explorar. Dado que (2b) y (2c) son desviaciones con respecto a la tendencia translingüística predominante, ¿es acaso posible que el factor causante de esas desviaciones sea el mismo? El atractivo que tiene esta idea es que, si fuese así, (2b) y (2c) podrían recibir un análisis unificado.

Para explicar la recurrencia de los inventarios nasales / $\mathrm{m},{ }^{*} \mathrm{n}, \mathrm{y} /$, $/ \mathrm{m},{ }^{*} \mathrm{n},{ }^{*} \mathrm{n} / \mathrm{y} / \mathrm{m},{ }^{*} \mathrm{n}, \mathrm{n} /$, proponemos un análisis basado en restricciones universales en el que se reconoce que ciertas restricciones de MARCACIÓN están sujetas a un orden jerárquico que también es universal. Valiéndonos de la teoría de optimidad (Prince y Smolensky 1993), demostramos que la composición del inventario nasal del español se puede explicar elegantemente por medio de la interacción entre una familia de restricciones de fidelidad y dos familias de restricciones de MARCACIÓN. Un atractivo adicional que tiene esta aproximación teórica es que explica los inventarios nasales de las lenguas como el inglés y el telugu simplemente por medio de variar el rango de las restricciones postuladas.

En el ámbito de la fidelidad, el principio universal que postulamos es la restricción FIEL(lugar nasal), la cual requiere la preservación de los rasgos de lugar de los fonemas nasales. En lo que respecta a la MARCACIÓN, adoptamos de Prince y Smolensky 1993 el esquema * [rasgo de lugar], el cual prohíbe la presencia de rasgos de lugar (p. ej. * [coronal], * [labial], *[dorsal], etc.) y motivamos un nuevo esquema que ciframos como * [nasal, rasgo de lugar], el cual prohíbe la combinación del rasgo de manera [nasal] con los diversos rasgos de lugar (p. ej. * [nasal, coronal,$*$ [nasal, labial], * [nasal, dorsal], etc.). Mediante la adopción de estos dos esquemas, nuestra propuesta nos provee de un recurso formal 
para que, no solamente la faceta de producción del habla -representada por * [rasgo de lugar]-, sino también la faceta de percepción del habla -representada por * [nasal, rasgo de lugar]- tengan un papel decisivo en la creación de inventarios fonémicos nasales.

Un supuesto esencial de este análisis es que los miembros de los esquemas * [rasgo de lugar] y * [nasal, rasgo de lugar] obedecen un orden jerárquico universal. Esto proporciona un criterio inequívoco para evaluar el coste gramatical implicado por la generación de CNPs con diferentes lugares de articulación. La predicción general es que, si el inventario nasal de una lengua incluye una CNP con un rasgo de lugar que viola una restricción de alto rango en una jerarquía universal de MARCACIÓN, entonces aquellas CNPs cuyos rasgos de lugar violen restricciones de menor rango en la misma jerarquía también estarán presentes, dado que su coste es menor. Inversamente, la ausencia de una CNP con un rasgo de lugar que viola una restricción de bajo rango en una jerarquía universal de MARCACIÓN implica que aquellas CNPs cuyos rasgos de lugar violen restricciones de mayor rango en la misma jerarquía también estarán ausentes, dado que su coste es mayor. En resumen, la presencia de CNPs marcadas implica la presencia de CNPs menos marcadas, pero no viceversa.

\section{EL MARCO TEÓRICO}

El propósito de esta sección es presentar brevemente las convenciones y supuestos básicos de la teoría de optimidad para beneficio de aquellos lectores que no estén familiarizados con este marco teórico. Para quienes lo estén, se recomienda pasar directamente a la sección 3.

Dentro de la teoría de optimidad (Prince y Smolensky 1993), se asume que la gramática de cada lengua consta de restricciones -principios lingüísticos universales- que se acatan con diferente orden de prioridad porque están organizadas jerárquicamente. Así, cuando dos restricciones entran en conflicto, aquella que tenga mayor rango se acatará a expensas de la que lo tenga menor. Dos clases de principio que chocan frecuentemente son las restricciones de FIDELIDAD -cuyo objetivo general es fomentar la identidad entre la entrada y la salida- y las restricciones de MARCACIÓN -cuyo objetivo general es evitar las estructuras complejas-. Para ilustrar el conflicto entre ellas, comparemos dos tipos de lengua, una que utiliza el inventario nasal /m, ${ }^{*} n, n,{ }^{*} n, n /$ y otra que emplea el inventario nasal $/{ }^{*} \mathrm{n} /$. Un representante del primer tipo de lengua es el burrara, perteneciente a la familia australiana, mientras que el segundo tipo lo ejemplifica el konkani, una lengua de la familia índica.

Para que un inventario nasal tan rico como el del burrara pueda surgir, es necesario que FIDELIDAD supere en rango a MARCACIÓN. Este 
orden de prioridad asegura que ninguna de las unidades que integran el inventario / $\mathrm{m},{ }^{*} \mathrm{n}, \mathrm{\eta},{ }^{*} \mathrm{n}, \mathrm{n} /$ se perderá. La obtención de este resultado se aprecia mejor en una tabla como la presentada en (3), donde se comparan las diferentes opciones lógicas que existen. Nótense las siguientes convenciones: la restricción dominante aparece a la izquierda de la restricción dominada y el símbolo $\gg$ se usa para indicar la relación de dominio. Las violaciones de las restricciones se indican a través de asteriscos. El signo de exclamación indica que la violación es fatal y que el candidato que incurre en ella es eliminado en ese punto. Las casillas a la derecha de una violación fatal aparecen sombreadas para indicar que son inconsecuentes. Por último, el candidato óptimo, o sea, aquel que respeta mejor las restricciones según el rango que tienen en la gramática, se identifica icónicamente por medio de una mano que señala.

\begin{tabular}{|c|c|c|}
\hline Entrada: $/ \mathrm{m}, * \mathrm{n}, \mathrm{y}, * \mathrm{n}, \mathrm{n} /$ & FidelidAD & MARCACIÓN \\
\hline a. $/ \mathrm{m}, * \mathrm{n}, \mathrm{y}, * \mathrm{n}, \mathrm{n} /$ & & $* * * * *$ \\
\hline b. $/ \mathrm{m}, * \mathrm{n}, \mathrm{y}, * \mathrm{n} /$ & $* !$ & $* * * *$ \\
\hline c. $/ \mathrm{m}, * \mathrm{n}, \mathrm{y} /$ & $* ! *$ & $* * *$ \\
\hline d. $/ \mathrm{m}, * \mathrm{n} /$ & $* ! * *$ & $* *$ \\
\hline e. $/ * \mathrm{n} /$ & $* ! * * *$ & $*$ \\
\hline
\end{tabular}

TABLA 3: FIDELIDAD >> MARCACIÓN.

En esta gramática, el candidato óptimo es (3a) debido a que, como FIDELIDAD domina a MARCACIÓN, es de suma importancia conservar los elementos de la entrada. Las violaciones se determinan de la siguiente manera: en vista de que la eliminación de nasales atenta contra la identidad entre la entrada y la salida, a aquellos candidatos que no preservan todas las unidades presentes en la entrada se les asigna un asterisco en la columna de FIDELIDAD por cada unidad suprimida (3b-d). De otra parte, como la preservación de nasales acarrea un coste estructural, a cada candidato se le asigna un asterisco en la columna de MARCACIÓN por cada unidad incluida. La aplicación de estos criterios revela que ninguno de los competidores consigue igualar al candidato (3a), el único que se mantiene completamente fiel a la entrada. La conclusión general que se desprende de esta evaluación es que, bajo la jerarquía FIDELIDAD $>$ MARCACIÓN, los inventarios nasales constituidos por un número abundante de unidades se ven favorecidos. 
Veamos ahora lo que sucede en una gramática en la que MARCACIÓN tiene prioridad frente a FIDELIDAD (4). Con esta inversión de prioridades, lo más importante ya no es la identidad entre la entrada y la salida, sino la evitación de estructuras complejas. A consecuencia de esto, el candidato que emerge victorioso ya no es el que replica mejor la entrada, sino aquel que opta por simplificar el inventario nasal suprimiendo las unidades más marcadas (4e). Es decir que el efecto general que causa la promoción de las restricciones de MARCACIÓn sobre las de FIDELIDAD es reducir el tamaño del inventario nasal.

\begin{tabular}{|c|c|c|}
\hline Entrada: $/ \mathrm{m}, * \mathrm{n}, \mathrm{\eta}, * \mathrm{n}, \mathrm{n} /$ & MARCACIÓN & FIDELIDAD \\
\hline a. $/ \mathrm{m}, * \mathrm{n}, \mathrm{y}, * \mathrm{n}, \mathrm{n} /$ & $* * ! * * *$ & \\
\hline b. $/ \mathrm{m}, * \mathrm{n}, \mathrm{y}, * \mathrm{n} /$ & $* * ! * *$ & $*$ \\
\hline c. $/ \mathrm{m}, * \mathrm{n}, \mathrm{y} /$ & $* * ! *$ & $* *$ \\
\hline d. $/ \mathrm{m}, * \mathrm{n} /$ & $* * !$ & $* * * *$ \\
\hline e. $/ * \mathrm{n} /$ & $*$ & $* * * *$ \\
\hline
\end{tabular}

TABLA 4: MARCACiÓN >> FidELIDAD.

Las lenguas que, como el inglés, el español y el telugu, emplean un inventario nasal integrado por tres unidades, representan un punto intermedio entre las gramáticas ilustradas por las tablas (3) y (4); o sea, un estado en el que la gramática no se inclina decididamente en favor de la Marcación ni tampoco de la Fidelidad. Para explicar por qué $/ \mathrm{m},{ }^{*} \mathrm{n}, \mathrm{y} /, / \mathrm{m},{ }^{*} \mathrm{n},{ }^{*} \mathrm{n} / \mathrm{y} / \mathrm{m},{ }^{*} \mathrm{n}, \mathrm{n} /$ son las ternas nasales favorecidas, tendremos que determinar cuáles son exactamente las restricciones de FIDELIDAD y de MARCACIÓN que están involucradas en el conflicto y cuáles con los rangos que las rigen en cada uno de esos tres sistemas gramaticales. Estos son los dos asuntos principales que se investigan a fondo en las siguientes secciones.

\section{LimitACIONES DE LA JERARQUía UNIVERSAL DE LUGAR}

Un fenómeno que sugiere que las lenguas reconocen diferencias de marcación entre rasgos de lugar es que, dentro de una misma clase consonántica, hay algunos lugares de articulación que son más comunes que otros. Es lógico suponer, por ejemplo, que el motivo por el que /*n/ aparece más frecuentemente en los inventarios nasales que / $\eta$ / es porque el lugar dental/alveolar es menos marcado que el retroflejo. Es- 
tructuralmente, la causa no podría ser otra más que esa en vista de que, aparte del lugar de articulación, estas dos CNPs coinciden en todos sus demás rasgos distintivos.

Asumir un orden jerárquico entre restricciones de marcación es una manera de evaluar el menor o mayor coste que tiene para la gramática adoptar ciertos rasgos de lugar. De acuerdo con (5), las restricciones de marcación que penalizan la presencia de rasgos de lugar obedecen un orden ascendente que va desde *[coronal], la restricción relevante para $/{ }^{*} \mathrm{n} /$, hasta * [sublaminal], la restricción pertinente para /n/. Entre estos dos extremos se encuentran tres rangos intermedios y gradualmente más altos representados por las restricciones *[labial], *[dorsal] $\mathrm{y} *[$ dorsal, coronal], las cuales conciernen a $/ \mathrm{m} /, / \mathrm{y} / \mathrm{y} / * \mathrm{n} /$, respectivamente.

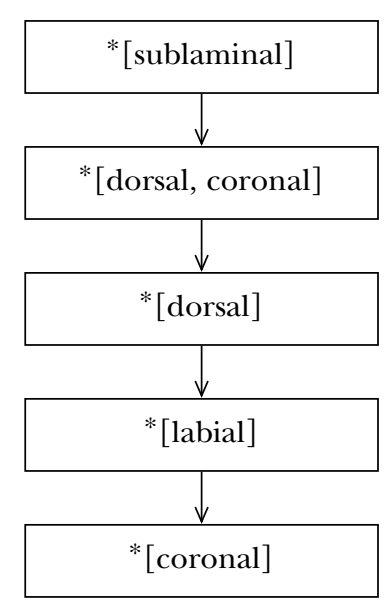

TABLA 5: Jerarquía articulatoria de lugar.

Con respecto a la mitad inferior de esta jerarquía, se ha propuesto ya en varios trabajos anteriores que los rasgos [coronal], [labial] y [dorsal] son gradualmente más marcados (Iverson y Kim 1987, Jun 1995, De Lacy 2002, entre otros). La posición adoptada aquí coincide con ese punto de vista, pero enfatiza que dicho orden depende críticamente de la dinámica de los gestos articulatorios, razón por la cual nos referimos a (5) como la «jerarquía articulatoria de lugar». La observación de gestos articulatorios sugiere que, en virtud de su composición neuromuscular, la corona es el articulador más apto para ejecutar el movimiento rápido y local necesario para producir consonantes, mientras que el labio inferior y el dorso, cuya sofisticación neuromuscular decrece inversamente 
al aumento de su masa, son gradualmente menos aptos para ese propósito. De conformidad con esto, a la restricción * [coronal] se le adjudica la posición más baja de la jerarquía, mientras que a las restricciones * [labial $\mathrm{y}^{*}$ [dorsal] se les asignan posiciones gradualmente más altas. El efecto que esto tiene es que la selección de consonantes coronales resulta menos costosa para la gramática que la selección de consonantes labiales, las cuales son a su vez menos costosas que las consonantes dorsales ${ }^{7}$.

La mitad superior de la «jerarquía articulatoria de lugar» incorpora el supuesto de que las categorías definidas por los rasgos [dorsal], [dorsal, coronal] y [sublaminal] también son gradualmente más marcadas. No es difícil concebir que una consonante cuya producción requiera la acción coordinada del dorso y la corona constituye un reto articulatorio más grande que otra que requiera la intervención del dorso solamente. Tampoco es insensato presumir que curvar la corona hacia atrás para que la sublámina pueda elevarse hacia el cielo de la boca y constreñir el tracto vocal impone un reto aun más grande a la habilidad motriz de la lengua. Aunque es innegable que estas estimaciones necesitan ser confirmadas por medio de estudios experimentales, es improbable que sean desacertadas dado que entre las lenguas de la UPSID encontramos evidencia que apunta en esa misma dirección.

La frecuencia con la que los diversos lugares de articulación figuran en los inventarios nasales recogidos en la UPSID provee una base empírica para las relaciones de marcación postuladas en (5). La tabla (6) muestra que el lugar retroflejo es el que con menor frecuencia figura en los inventarios de CNPs. Solamente $5.32 \%$ de las lenguas de la UPSID tienen CNPs con ese lugar. En cambio, las CNPs con lugar palatal $(41.24 \%)$, velar $(52.55 \%)$, bilabial $(94.24 \%)$ y dental/alveolar $(98.67 \%)$ ocurren con una frecuencia que supera el $40 \%$ y aumenta gradualmente en un orden que coincide exactamente con el orden descendente especificado por la «jerarquía articulatoria de lugar» ${ }^{8}$.

7 Asumimos que las consonantes glotales $-/ \mathrm{h} / \mathrm{y} / \mathrm{P} / \mathrm{-}$ son segmentos que carecen de un rasgo específico de lugar, una situación que las hace más marcadas que otras consonantes porque lo normal es que cada segmento incluya su propio rasgo de lugar. Es decir, que, en cuanto a la marcación, las consonantes glotales violan mínimamente la restricción TENERLUGAR.

8 Admitimos que es probable que la «jerarquía articulatoria de lugar» incluya otras restricciones por encima de *[sublaminal]. Es razonable suponer, por ejemplo, que *[labial, dorsal], es decir, la restricción pertinente para las consonantes labiovelares, domina a * [sublaminal]. La observación que sugiere que esto es así es que la producción de labiovelares involucra dos articuladores, ninguno de los cuales es el más apto para desplazarse a gran velocidad (cf. /*n/). Otra evidencia en este sentido es que la frecuencia con la que la CNP labiovelar, / $\overparen{\mathrm{my}} /$, aparece en los inventarios nasales de las lenguas de la UPSID es menor que la de la retrofleja / $\mathrm{n} /$ ( $1.55 \%$ y $5.32 \%$, respectivamente). A pesar de estas consideraciones, la restricción *[labial, dorsal] se ha dejado por fuera de (5) porque, como la doble constricción oral que caracteriza a $/ \overparen{\mathrm{my}} /$ obliga a clasificarla no como una CNP sino como una CNS, la restricción de marcación que atañe a ese fonema es irrelevante para la presente discusión. 


\begin{tabular}{|l|c|l|c|}
\hline \multicolumn{1}{|c|}{ Lugar } & Símbolo & \multicolumn{1}{c|}{ Rasgos } & Porcentaje \\
\hline Retroflejo & $/ \mathrm{n} /$ & [sublaminal] & $5.32 \%$ \\
\hline Palatal & $/ * \mathrm{n} /$ & [dorsal, coronal] & $41.24 \%$ \\
\hline Velar & $/ \mathrm{n} /$ & [dorsal] & $52.55 \%$ \\
\hline Bilabial & $/ \mathrm{m} /$ & [labial] & $94.24 \%$ \\
\hline Dental/alveolar & $/ * \mathrm{n} /$ & [coronal] & $98.67 \%$ \\
\hline
\end{tabular}

TABLA 6: Frecuencia de CNPs en la UPSID según su lugar de articulación.

Asumir que la jerarquía propuesta en (5) es universal es de gran interés teórico, porque predice ciertas relaciones de implicación. En general, la presencia de una CNP que posea un rasgo de lugar, prohibido por una restricción alta en la jerarquía, implica que las CNPs que posean rasgos de lugar prohibidos por restricciones más bajas también estarán presentes, debido a que el coste que la gramática tiene que pagar por ellas es menor. Para dar un ejemplo concreto, si el inventario fonémico de una lengua incluye una CNP con el rasgo [dorsal], se espera que tal lengua también tenga una CNP con el rasgo [labial] y otra con el rasgo [coronal]. Es decir que la presencia de / $\mathrm{y} / \mathrm{implica} q u e / \mathrm{m} / \mathrm{y} /{ }^{*} \mathbf{n} /$ también estarán presentes. Eso es justamente lo que encontramos en las lenguas que, como el inglés, usan la terna $/ \mathrm{m},{ }^{*} \mathrm{n}, \mathrm{y} /$. Este inventario nasal refleja transparentemente el orden de dominio especificado por la mitad inferior de la «jerarquía articulatoria de lugar».

Dentro de la teoría de optimidad (Prince y Smolensky 1993), la gramática de una lengua que emplea el inventario nasal /m, *n, y/ se puede modelar al permitir que la restricción de fidelidad que protege los rasgos de lugar de las consonantes nasales, Fiel(lugar nasal), interrumpa la jerarquía de lugar en un punto medio. Más específicamente, FiEL(lugar nasal) debe tener un rango superior a *[dorsal], pero inferior a *[dorsal, coronal], como se ilustra en el diagrama (7).

La dominancia de FIEL(lugar nasal) sobre * [dorsal] garantiza que una CNP cuyo rasgo de lugar sea igual o menos marcado que [dorsal] se conservará intacta en esta gramática. Esto queda demostrado en las tablas (8A-C), donde el candidato elegido como la salida óptima es idéntico a la forma de entrada (8Aa, 8Bb y 8Cc). La dominancia de *[dorsal, coronal] sobre FIEL (lugar nasal) asegura, por otra parte, que ninguna CNP que incluya un rasgo de lugar igual o más marcado que la combinación [dorsal, coronal] sobrevivirá. La tabla (8D) demuestra que, en ese caso, la salida óptima es $/{ }^{*} \mathrm{n} /$ porque, una vez que la preservación de un ras- 


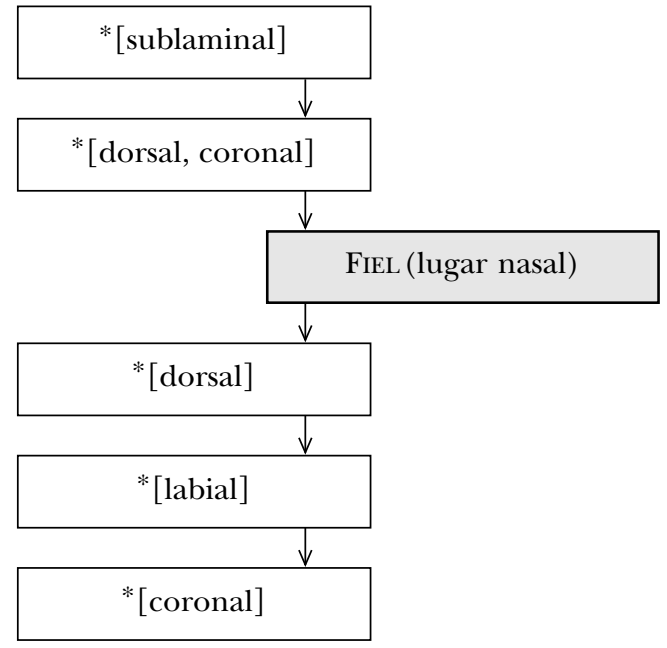

TABLA 7: Gramática de las lenguas con la terna /m, *n, y/ (versión preliminar).

go de lugar excede el umbral protegido por la restricción de fidelidad relevante, la decisión pasa a depender de la «jerarquía articulatoria de lugar», la cual favorece el rasgo de lugar menos marcado de todos (8Da). Si bien es cierto que el sistema de restricciones presentado en (7) produce resultados positivos en el caso de las lenguas como el inglés,

A.

\begin{tabular}{|r|l|c|c|c|c|c|}
\hline \multicolumn{2}{|c|}{ Entrada: $/ * \mathrm{n} /$} & $*[$ dor, cor $]$ & FIEL(lug nas) & $*[$ dor $]$ & $*[$ lab $]$ & $*[$ cor $]$ \\
\hline a. & $/ * \mathrm{n} /$ & & & & & $*$ \\
\hline b. & $/ \mathrm{m} /$ & & $* !$ & & $*$ & \\
\hline $\mathrm{c}$. & $/ \mathrm{y} /$ & & $* !$ & $*$ & & \\
\hline
\end{tabular}

B.

\begin{tabular}{|r|l|c|c|c|c|c|}
\hline \multicolumn{2}{|c|}{ Entrada: $/ \mathrm{m} /$} & $*[$ dor, cor $]$ & FIEL(lug nas) & $*[$ dor $]$ & $*[$ lab $]$ & $*[$ cor $]$ \\
\hline a. & $/ * \mathrm{n} /$ & & $* !$ & & & $*$ \\
\hline b. & $/ \mathrm{m} /$ & & & & $*$ & \\
\hline c. & $/ \mathrm{y} /$ & & $* !$ & $*$ & & \\
\hline
\end{tabular}


C.

\begin{tabular}{|r|l|c|c|c|c|c|}
\hline \multicolumn{2}{|c|}{ Entrada: $/ \mathrm{y} /$} & $*[$ dor, cor $]$ & FIEL(lug nas) & $*[$ dor $]$ & $*[$ lab $]$ & $*[$ cor $]$ \\
\hline a. & $/ * \mathrm{n} /$ & & $* !$ & & & $*$ \\
\hline $\mathrm{b}$. & $/ \mathrm{m} /$ & & $* !$ & & $*$ & \\
\hline $\mathrm{c}$. & $/ \mathrm{y} /$ & & & $*$ & & \\
\hline
\end{tabular}

D.

\begin{tabular}{|r|c|c|c|c|c|c|}
\hline \multicolumn{2}{|c|}{ Entrada: $/ * \mathrm{n} /$} & $*[$ dor, cor $]$ & FIEL(lug nas) & $*[$ dor $]$ & $*[$ lab $]$ & $*[$ cor $]$ \\
\hline a. & $/ * \mathrm{n} /$ & & $*$ & & & $*$ \\
\hline $\mathrm{b}$. & $/ \mathrm{m} /$ & & $*$ & & $* !$ & \\
\hline $\mathrm{c}$. & $/ \mathrm{y} /$ & & $*$ & $* !$ & & \\
\hline $\mathrm{d}$. & $/ * \mathrm{n} /$ & $* !$ & & & & \\
\hline
\end{tabular}

TABLA 8: Generación de la terna / $\mathrm{m}, * \mathrm{n}, \mathrm{y} /$ en una lengua como el inglés.

los resultados son mucho menos satisfactorios cuando intentamos usar esas mismas restricciones para generar los inventarios nasales de lenguas como el español o el telugu. El intento de derivar las ternas nasales /m, *n, *n/ y /m, *n, n/ variando la posición de FIEL(lugar nasal) con respecto a los integrantes de la «jerarquía articulatoria de lugar»es fallido porque, para que la gramática pueda generar $/{ }^{*} \mathrm{n} / \mathrm{o} / \mathrm{n} /$, sería necesario que FIEL(lugar nasal) tuviera un rango más alto que el que se le adjudicó en (7). Desafortunadamente, una consecuencia inevitable de realizar tal ajuste es que la gramática resultante generaría también todas aquellas CNPs que posean rasgos de lugar menos marcados que los que caracterizan a $/ * \mathrm{n} / \mathrm{y} / \mathrm{n} /$.

El problema se aprecia claramente en (9) y (10). Obsérvese que una vez que Fiel(lugar nasal) es promovida por encima de * [dorsal, coronal], el fonema /*n/ resulta admitido; sin embargo, el efecto global no es exactamente el que buscamos, dado que el inventario generado por tal gramática no es la terna $/ \mathrm{m},{ }^{*} \mathrm{n},{ }^{*} \mathrm{n} /$, sino el cuarteto $/ \mathrm{m},{ }^{*} \mathrm{n}, \mathrm{y},{ }^{*} \mathrm{n} /(9)$. Similarmente, cuando se permite que Fiel(lugar nasal) ascienda por encima de *[sublaminal], el fonema $/ \mathrm{n} /$ se ve incluido, pero el resultado global tampoco es el deseado porque el inventario que tal gramática genera no es la terna $/ \mathrm{m},{ }^{*} \mathrm{n}, \mathrm{\eta} /$, sino el quinteto /m, *n, $\mathrm{y},{ }^{*} \mathrm{n}, \mathrm{\eta} /(10)$. En breve, la estrategia de variar el rango de FIEL(lugar nasal) con respecto a la «jerarquía articulatoria de lugar» funciona bien para controlar 


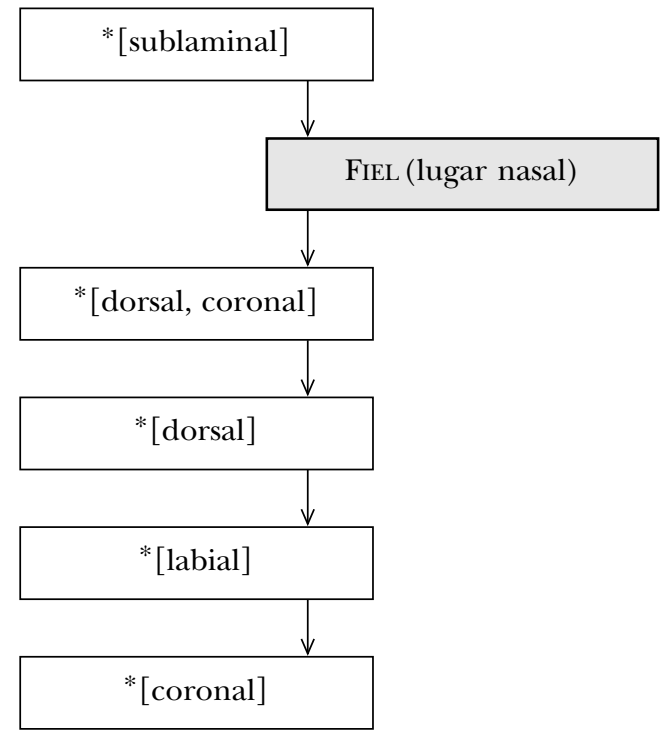

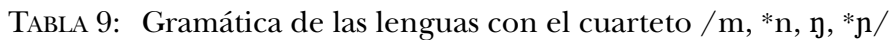
(versión preliminar).

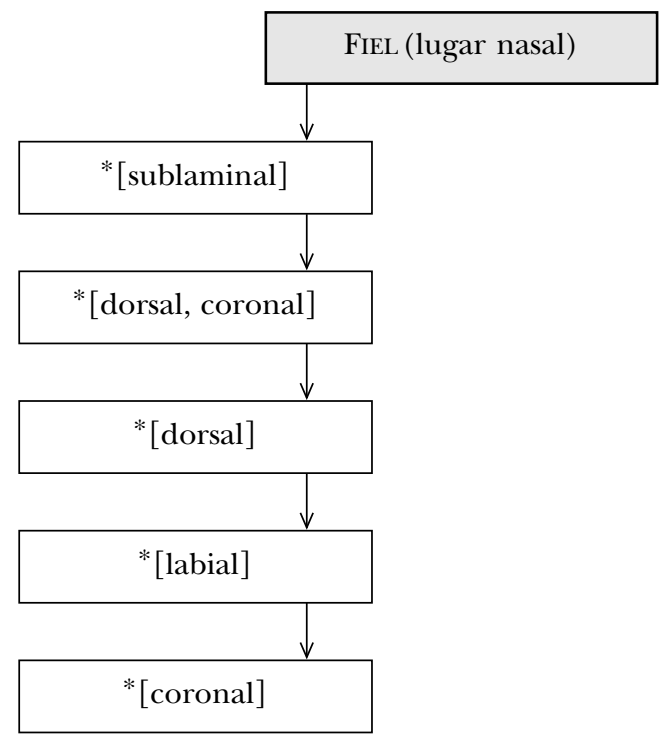

TABLA 10: Gramática de las lenguas con el quinteto /m, *n, y, *n, n/ (versión preliminar). 
el tamaño de los inventarios nasales, pero no para generar las ternas $/ \mathrm{m},{ }^{*} \mathrm{n},{ }^{*} \mathrm{n} / \mathrm{y} / \mathrm{m},{ }^{*} \mathrm{n}, \mathrm{n} /$ como alternativas al inventario /m, *n, $\mathrm{y} /$.

En vista de que la promoción de rango de FIEL(lugar nasal) no produce los resultados deseados, la otra ruta obvia que podríamos explorar es alterar el orden fijo de algunos de los miembros de la «jerarquía articulatoria de lugar». Serían específicamente las restricciones *[sublaminal ], * [dorsal, coronal] y * [dorsal] las que tendrían que manipularse. Las lenguas que seleccionan $/{ }^{*} \mathrm{n} /$ como la tercera CNP requerirían reformar la jerarquía de modo que *[dorsal] y*[dorsal, coronal] invirtiesen el orden, como lo indica la flecha bidireccional en (11A $)^{9}$. Las lenguas que seleccionan / $\mathrm{n} /$ como la tercera CNP requerirían, por otro lado, que el orden entre *[dorsal] y*[sublaminal] se invirtiese, como se indica en (11B) por medio de la flecha bidireccional. Adicionalmente, sería necesario asumir que FiEL(lugar nasal) mantiene una posición constante entre el tercero y el cuarto nivel de la jerarquía de lugar. Así, las gramáticas resultantes, (11A) y (11B), tendrían éxito en generar los inventarios $/ \mathrm{m},{ }^{*} \mathrm{n},{ }^{*} \mathrm{n} / \mathrm{y} / \mathrm{m},{ }^{*} \mathrm{n}, \mathrm{n} /$ sin generar simultáneamente ninguna otra CNP.

A. Inventario $/ \mathrm{m}, * \mathrm{n}, * \mathrm{n} /$

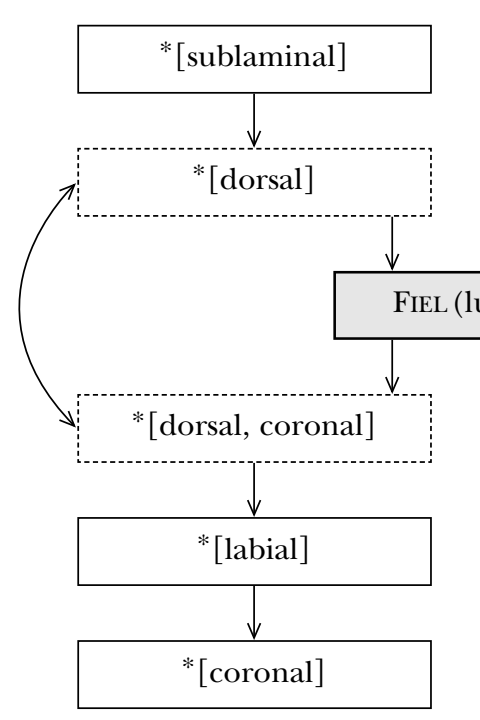

B. Inventario $/ \mathrm{m}, * \mathrm{n}, \mathrm{n} /$

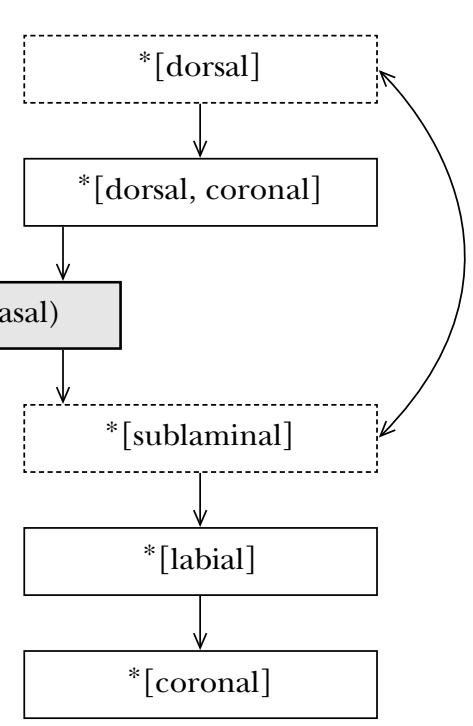

TABLA 11: Variaciones en la jerarquía de lugar para obtener las ternas nasales alternativas.

\footnotetext{
${ }^{9}$ Para resaltarlas, las restricciones cuyo orden ha sido alterado con respecto a la posición que ocupan en (7) aparecen en cuadros dibujados con líneas discontinuas.
} 
A pesar de su capacidad para generar las ternas nasales que buscamos, la alternativa teórica que acabamos de discutir está basada en un razonamiento que es excesivamente permisivo. Si se permitiera que dos o más restricciones de la «jerarquía articulatoria de lugar» permutasen su orden, se tendría que aceptar que todos los demás integrantes de dicha jerarquía también cambiaran de orden, lo cual tendría consecuencias desastrosas. En el caso de las lenguas que emplean una sola CNP se esperaría, por ejemplo, que el fonema $/ \mathrm{n} /$, el cual posee un rasgo de lugar altamente marcado, fuese la CNP elegida por encima de otras CNPs que tienen rasgos de lugar menos marcados (p. ej. /m/ y /*n/). Esto sería posible porque, si el orden entre los miembros de la «jerarquía articulatoria de lugar» fuese permutable, la restricción * [sublaminal] podría ser ubicada por debajo de todos los demás integrantes de esa jerarquía convirtiéndola así en la CNP óptima. Para las lenguas que forman sus inventarios nasales por medio de contrastar tres CNPs se predeciría que no solamente /m, *n, $\mathrm{y} /, / \mathrm{m},{ }^{*} \mathrm{n},{ }^{*} \mathrm{n} / \mathrm{y} / \mathrm{m},{ }^{*} \mathrm{n}, \mathrm{n} /$ sino también las demás ternas que se pueden obtener por medio de combinar los fonemas involucrados sirvieran como inventarios nasales en ciertas lenguas.

La realidad es, sin embargo, que tal estado de cosas no existe. La UPSID evidencia que ninguna de las lenguas incluidas allí utiliza CNPs altamente marcadas como su único fonema nasal -por ejemplo, /n/, $/{ }^{*} \mathrm{n} /, \mathrm{o} / \mathrm{y} /-\mathrm{y}$ que la mayoría de las ternas nasales que resultan de hacer combinaciones lógicas de CNPs -por ejemplo, /n, *n, $\mathrm{y} /, / \mathrm{n},{ }^{*} \mathrm{n}, \mathrm{m} /$, $/ \mathrm{n}, \mathrm{\eta}, \mathrm{m} /, / \mathrm{n},{ }^{*} \mathrm{n},{ }^{*} \mathrm{n} /, / \mathrm{n}, \mathrm{\eta},{ }^{*} \mathrm{n} /, /{ }^{*} \mathrm{n}, \mathrm{\eta},{ }^{*} \mathrm{~m} / \mathrm{y} /{ }^{*} \mathrm{n}, \mathrm{y},{ }^{*} \mathrm{n} /-$ tampoco se verifican como inventarios nasales en ninguna lengua. La conclusión que extraemos de esto es que la libre permutación de rangos entre los miembros de la «jerarquía articulatoria de lugar» debe rechazarse categóricamente porque equivaldría a ubicar todas las restricciones contra los rasgos de lugar en un mismo nivel. El gran inconveniente que esto tiene es que, sin una ordenación jerárquica entre los principios relevantes, las relaciones de implicación entre CNPs serían un absoluto misterio.

Frente al hecho irrebatible de que los inventarios nasales no se crean fortuitamente sino que hay ciertas CNPs y ciertas combinaciones de CNPs que priman sobre otras, el camino teórico más viable que podemos tomar es respetar estrictamente el supuesto de que la «jerarquía articulatoria de lugar» tiene carácter universal, lo cual es congruente con la base articulatoria de la que hemos partido. A este respecto, es pertinente apuntar que, por encima de las diferencias de raza, los seres humanos disponemos de los mismos órganos para la producción de sonidos lingüísticos, con lo cual las capacidades y limitaciones del aparato fonador son las mismas para toda la especie sin importar la lengua específica que se hable. Lo que esto sugiere es que la universalidad que se le atribuye aquí a la «jerarquía articulatoria de lugar» es una propiedad que el componente fonético impone sobre el componente fonológico. 
En síntesis, en esta sección hemos probado que, aunque existe evidencia en favor de una «jerarquía articulatoria de lugar» de carácter universal, la interacción entre las restricciones que integran esa jerarquía y la restricción de fidelidad que protege los rasgos de lugar de las consonantes nasales no es una estrategia satisfactoria para derivar las ternas nasales $/ \mathrm{m},{ }^{*} \mathrm{n},{ }^{*} \mathrm{n} / \mathrm{y} / \mathrm{m},{ }^{*} \mathrm{n}, \mathrm{n} /$, las cuales surgen en algunas lenguas como alternativas al inventario $/ \mathrm{m}, * \mathrm{n}, \mathrm{y} /$. Apelar a permutaciones de rango entre los miembros de la «jerarquía articulatoria de lugar» es una forma de forzar la gramática a que produzca los resultados deseados; sin embargo, argüimos que los análisis que recurran a tal estrategia deben rechazarse porque están basados en un razonamiento que permite generar inventarios nasales inexistentes y fallan en reconocer las relaciones de implicación que existen entre CNPs.

\section{EL PAPEL DE LA PERCEPCIÓN EN LA FORMACIÓN DE INVENTARIOS NASALES}

En esta sección nos proponemos motivar la propuesta de que, además de las restricciones de marcación que penalizan los rasgos de lugar según la habilidad motriz de los articuladores que los implementan, existen también restricciones de marcación que penalizan los rasgos de lugar según la ubicación de la constricción oral con respecto al puerto velofaríngeo. Resulta que, por motivos perceptivos que se explicarán más abajo, cuanto menor sea la distancia entre la constricción oral y el puerto velofaríngeo, más costosa es la adopción del rasgo de lugar responsable de tal constricción. Para formalizar este fenómeno, que es una idiosincrasia de las consonantes nasales, proponemos una jerarquía universal de perceptibilidad nasal, cuya interacción con la jerarquía universal de lugar y la restricción de fidelidad que protege los rasgos de lugar de las consonantes nasales ofrece una manera satisfactoria y elegante de derivar los inventarios nasales $/ \mathrm{m},{ }^{*} \mathrm{n}, \mathrm{y} /, / \mathrm{m},{ }^{*} \mathrm{n},{ }^{*} \mathrm{n} / \mathrm{y} / \mathrm{m},{ }^{*} \mathrm{n}, \mathrm{n} /$.

El hecho de que existan dos grupos minoritarios de lenguas que, en vez de completar sus ternas nasales con una CNP velar, optan por una CNP palatal o retrofleja nos incita a considerar la posibilidad de que $/{ }^{*} \mathrm{n} / \mathrm{y} / \mathrm{n} /$ tengan cierta ventaja sobre $/ \mathrm{n} /$. El valor de explorar este asunto es que, si encontrásemos algún aspecto estructural en el que $/ \mathrm{n} /$ aparezca en desventaja con respecto a $/{ }^{*} \mathrm{n} / \mathrm{o} / \mathrm{\eta} /$, ello supondría que existe una dimensión en la que /y/ es más marcada, lo cual podría explicar por qué algunas lenguas optan por usar ternas nasales que la excluyen. Para avanzar en esta dirección, conviene contrastar los diagramas del tracto vocal que aparecen en (12), donde se puede apreciar que una consecuencia directa de que $/ \mathrm{y} /, /{ }^{*} \mathrm{n} / \mathrm{y} / \mathrm{n} /$ ocluyan el tracto vocal en diferentes puntos de la cavidad oral es que surgen diferencias considerables en la longitud de ese resonador. 


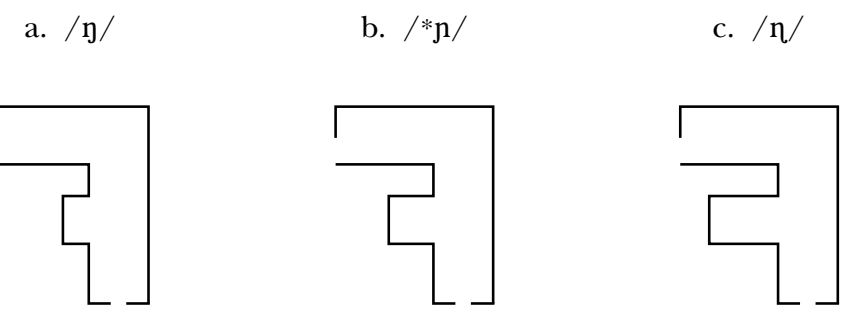

TABLA 12: Diferencias entre $/ \mathrm{y} /, / * \mathrm{n} / \mathrm{y} / \mathrm{n} /$ con respecto a la longitud de la cavidad oral.

En (12a-c), los resonadores que forman el tracto vocal -la cavidad faríngea, la cavidad nasal y la cavidad oral- se representan como conductos vacíos delimitados por líneas. En cada uno de esos diagramas, la apertura que aparece en el extremo izquierdo superior corresponde a las fosas nasales, mientras que la apertura que aparece en el extremo derecho inferior corresponde a la glotis. Lo que tienen en común las tres configuraciones es que la longitud del conducto formado por la cavidad faríngea y la cavidad nasal permanece constante, mientras que el aspecto en el que hay una clara divergencia es que la longitud del conducto formado por la cavidad oral aumenta significativamente al pasar $\mathrm{de} / \mathrm{y} / \mathrm{a} /{ }^{*} \mathrm{n} / \mathrm{y}$ de esta a /n/. Aunque la cavidad oral no funciona como el resonador primario en la articulación de las consonantes oclusivas nasales -ese papel lo desempeña el conducto faringo-nasal-, las diferencias en la longitud de este resonador son pertinentes porque producen un efecto acústico notable que discutiremos a continuación.

Los diagramas de (13) son idénticos a los de (12), excepto en que se han añadido flechas para indicar la dirección que sigue el flujo de aire en la producción de $/ \mathrm{y} /, /{ }^{*} \mathrm{n} / \mathrm{y} / \mathrm{n} /$. El primer conducto por el que pasa la corriente de aire proveniente de la glotis es la faringe, donde no encuentra ningún impedimento que le oponga resistencia. Al encontrar el puerto velo-faríngeo abierto, la corriente de aire puede entrar en la cavidad nasal y transitar libremente por allí hasta su salida hacia el exterior a través de las fosas nasales. En el curso de este recorrido, sin embargo, una parte del flujo que transita por la faringe se desborda hacia la cavidad oral, en donde la oclusión formada por los labios, la corona, o el dorso actúa como una barrera que impide el escape a través de la boca. La corriente secundaria que ello crea en la cavidad oral avanza tanto como la ubicación de la oclusión lo permite; no puede escapar por la boca porque la barrera creada por la oclusión hace que el aire rebote hacia la faringe, de modo que vuelve a unirse a la corriente principal, como lo indica la dirección de las flechas en los diagramas. 


\section{a. $/ \mathrm{y} /$}

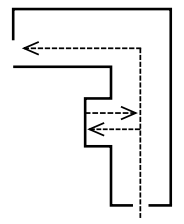

b. $/ * \mathrm{n} /$

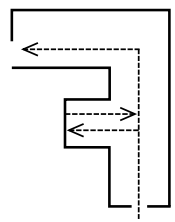

c. $/ \mathrm{n} /$

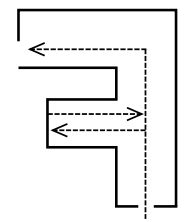

TABLA 13: Flujo de aire a través de los resonadores en $/ \mathrm{y} /, / * \mathrm{n} / \mathrm{y} / \mathrm{n} /$.

Gracias al flujo de aire que acabamos de describir, las oclusivas nasales tienen propiedades acústicas que las identifican inequívocamente. La corriente de aire principal aparece en el espectro acústico en forma de frecuencias faringo-nasales amplificadas -resonancias-, mientras que la corriente de aire secundaria se refleja en el espectro acústico en forma de frecuencias orales atenuadas -anti-resonancias-. Las resonancias faringo-nasales y las anti-resonancias orales generan el efecto acústico conocido como murmullo nasal. Así, las consonantes nasales se distinguen de las demás clases consonánticas por ser las únicas que cuentan con murmullo nasal.

Dado que el tamaño del conducto faringo-nasal es esencialmente el mismo para todas las oclusivas nasales, no existen diferencias considerables entre ellas en cuanto a las resonancias. Sin embargo, como el tamaño de la cavidad oral varía significativamente de una oclusiva nasal a otra, sí existen diferencias considerables en cuanto a las anti-resonancias. Esa es la razón de que, aunque todas las oclusivas nasales poseen murmullo nasal, la cualidad del murmullo no es idéntica en todas ellas. Esto es significativo porque se ha descubierto que, además de las transiciones de las vocales adyacentes, el murmullo nasal es una de las pistas acústicas que contribuye a la percepción e identificación del lugar de articulación (Malécot 1956, Fujimora 1962, Nord 1976, Recasens 1983, Kurowski y Blumstein 1984, 1987, 1993, Massone 1988, Albalá 1992, entre otros). Se encontró, por ejemplo que, al manipular la señal acústica suprimiendo las transiciones vocálicas y conservando el murmullo nasal, los aciertos son mejores que lo que se esperaría si fuesen al azar. Además, cuando los estímulos incluyen las transiciones vocálicas junto con el murmullo nasal, el número de aciertos es mayor que cuando se usan estímulos que incluyen solo las transiciones vocálicas.

Se ha descubierto que las anti-resonancias que aparecen en el espectro acústico de las CNPs varían inversamente a la longitud de la cavidad oral (House 1957, Ohala 1975, Ohala y Ohala 1993). Resulta que, 
cuanto más se reduce la longitud de ese resonador, más alta es la frecuencia de las anti-resonancias. En los espectros acústicos de /n/, /*n/ $\mathrm{y} / \mathrm{y} /$, por ejemplo, se detectan anti-resonancias cuya frecuencia es gradualmente más alta a causa de que estos fonemas usan una cavidad oral gradualmente más corta (13). Entonces, si agregamos a este grupo las otras dos CNPs más usadas, /*n/ y /m/, se obtiene el continuo presentado en (14), donde / $\mathrm{y} /$ queda ubicada en el extremo superior por poseer las anti-resonancias más altas, mientras que $/ \mathrm{m} /$ ocupa el extremo inferior por poseer las anti-resonancias más bajas ${ }^{10}$.

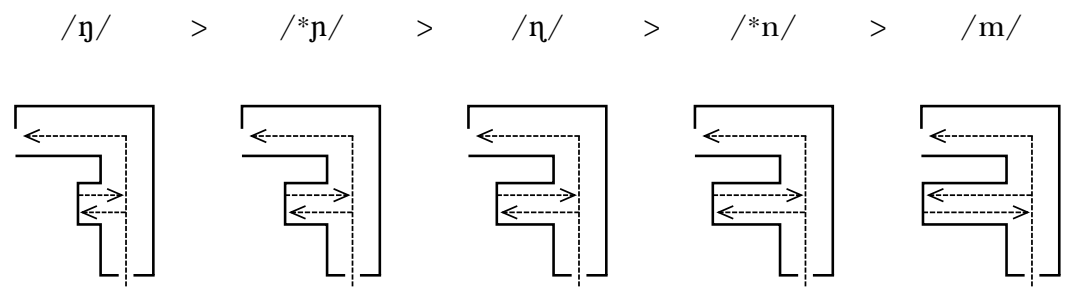

TABLA 14: Continuo de CNPs según la frecuencia de sus anti-resonancias.

Desde el punto de vista perceptivo, gravitar hacia el extremo superior del continuo en (14) no constituye una ventaja sino una desventaja. En todos los sonidos sonoros, la concentración de energía disminuye a medida que la frecuencia aumenta, lo cual perjudica a las CNPs articuladas en la parte posterior de la cavidad oral, ya que, como sus antiresonancias son de alta frecuencia, estas van a aparecer precisamente en el área del espectro donde la energía se ve más atenuada (House 1957, Ohala 1975, Ohala y Ohala 1993). La disminución de energía en las altas frecuencias perjudica menos a las CNPs articuladas en la parte anterior de la cavidad oral porque, como sus anti-resonancias son de menor frecuencia, aparecen en áreas del espectro donde todavía hay bastante concentración de energía.

Tales hallazgos en el campo acústico son congruentes con los resultados de una serie de experimentos de percepción realizados recientemente por Johnson, DiCanio y MacKenzie 2007. Este grupo de investigadores concluye que los oyentes que participaron en sus experimentos

\footnotetext{
${ }^{10}$ Obviamente, si se incluyera la uvular /N/, la cual se ha dejado de lado porque su frecuencia es extremadamente baja $(0.22 \%)$, es a esta a la que habría que adjudicar la posición más alta del continuo, ya que es la consonante nasal más posterior que existe. En virtud de esa propiedad, también es la que posee las anti-resonancias más altas.
} 
confundieron el estímulo de una vocal nasal con una secuencia de vocal seguida por /y/ mucho más frecuentemente que con una secuencia de vocal seguida por $/ \mathrm{n} / \mathrm{o} / \mathrm{m} /$. La tendencia a interpretar la nasalidad de una vocal nasal como una $/ \mathrm{y} /$ siguiente se deriva naturalmente del hecho de que las anti-resonancias de alta frecuencia que aparecen en el espectro de las consonantes nasales posteriores no son sobresalientes, por lo que se pueden confundir fácilmente con la ausencia de antiresonancias característica del espectro de las vocales nasales. Es menos probable que las consonantes nasales anteriores se vean involucradas en tal confusión porque sus anti-resonancias de baja frecuencia son más notables y aseguran un mejor contraste con respecto al espectro de una vocal nasal.

La conclusión que se desprende de las anteriores observaciones es que, cuanto más posterior sea la oclusión oral de una CNP, menos prominentes serán sus anti-resonancias y, por consiguiente, más difícil será la percepción de su lugar de articulación. De acuerdo con esto, la «jerarquía de perceptibilidad nasal» propuesta en (15) le adjudica un rango alto a las restricciones de marcación que prohíben la combinación del rasgo [nasal] con aquellos rasgos de lugar causantes de constricciones posteriores. En contraste, a las restricciones de marcación que prohíben la combinación del rasgo [nasal] con rasgos de lugar causantes de constricciones anteriores se les atribuye un rango inferior. Esto significa que, desde el punto de vista perceptivo, /m/ es la CNP más económica que las lenguas humanas tienen a su disposición. Las demás CNPs implican un coste perceptivo gradualmente mayor.

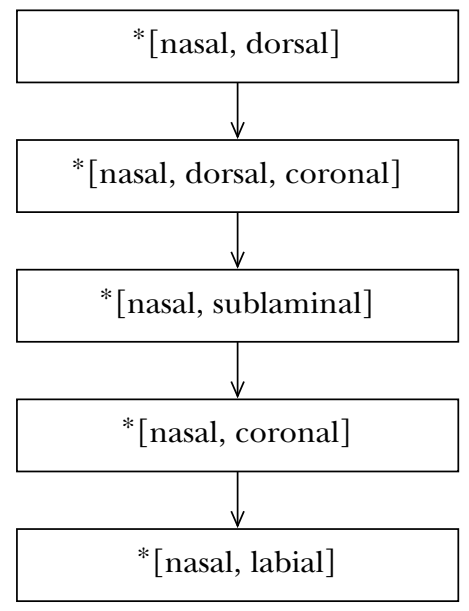

TABLA 15: Jerarquía de perceptibilidad nasal. 
Para demostrar el carácter universal de la jerarquía en (15), empezaremos por considerar las repercusiones que esta tiene para la derivación de inventarios nasales compuestos por tres unidades. Las lenguas que, como el inglés, eligen la velar / $\mathrm{y} /$ para completar su terna nasal, obedecen al sistema de restricciones dado en (16), el cual es una ampliación de (7). Tales gramáticas le conceden prioridad a Fiel (lugar nasal) sobre todas las restricciones de marcación que evalúan las CNPs según su coste perceptivo, pero la ubican en un rango intermedio con respecto a las restricciones de marcación que evalúan las CNPs según su coste articulatorio. Son específicamente las siguientes relaciones las que son cruciales: * [dorsal, coronal] $>$ FIEL (lugar nasal) $>$ * [nasal, dorsal]. No hay necesidad de especificar el rango de FIEL(lugar nasal) con respecto a cada uno de los miembros de las dos familias de marcación porque, una vez que su rango con respecto a una de esas restricciones queda establecido, los rangos con respecto a los demás miembros se derivan de las jerarquías universales propuestas en (5) y (15). Para dar ejemplos

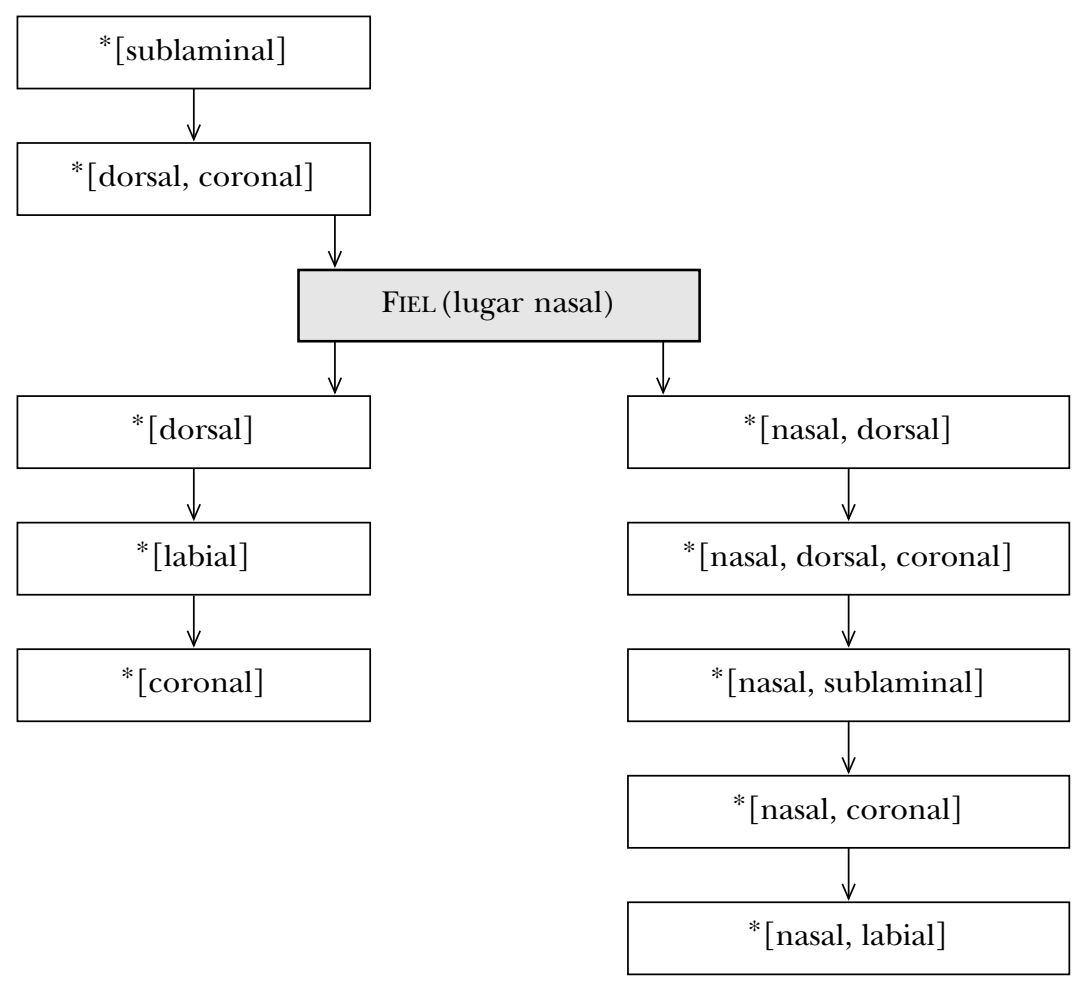

TABLA 16: Gramática de las lenguas con la terna /m, *n, y/ (versión final). 
concretos, si se establece que FIEL(lugar nasal) domina a * [nasal, dorsal], eso implica que también está por encima de *[nasal, dorsal, coronal] y todos los demás miembros de esa familia. Similarmente, si se establece que, por otro lado, Fiel (lugar nasal) está dominada por *[dorsal, coronal], eso implica que también está por debajo de * [sublaminal].

El poder generativo de esta gramática se demuestra en las tablas (17AD). Cuando la forma subyacente es una CNP con un rasgo de lugar de menor o igual coste articulatorio que [dorsal] $-\mathrm{o}$ sea, $/{ }^{*} \mathrm{n} /, / \mathrm{m} / \mathrm{o} / \mathrm{y} /-$, la salida óptima es aquella que conserva esa propiedad intacta (17Aa, $17 \mathrm{Bb}$ y $17 \mathrm{Cc}$ ). En contraste, si se postula una CNP con un rasgo de lugar articulatoriamente más marcado que [dorsal], la salida óptima resulta ser [n], o sea, la oclusiva nasal que, desde el punto de vista articulatorio, es la menos marcada de todas (17Da). Para evaluar este caso, es preciso incluir en la tabla la restricción *[labial] a fin de que se pueda apreciar por qué $/ \mathrm{m} /$ es articulatoriamente menos deseable que $/{ }^{*} \mathrm{n} /(17 \mathrm{Db})$.

El lector puede verificar que estos resultados son exactamente los mismos que los obtenidos en (8) y la razón es evidente. Como las len-

A.

\begin{tabular}{|r|c|c|c|c|c|}
\hline \multicolumn{2}{|c|}{ Entrada: $/ * \mathrm{n} /$} & $*[$ dor, cor] & FIEL(lug nas) & $*[$ nas, dor $]$ & $*[$ dor $]$ \\
\hline a. & $/ * \mathrm{n} /$ & & & & \\
\hline b. & $/ \mathrm{m} /$ & & $* !$ & & \\
\hline c. & $/ \mathrm{y} /$ & & $* !$ & $*$ & $*$ \\
\hline
\end{tabular}

B.

\begin{tabular}{|r|c|c|c|c:c|}
\hline \multicolumn{2}{|c|}{ Entrada: $/ \mathrm{m} /$} & $*$ [dor, cor] & FIEL(lug nas) & $*$ [nas, dor] & $*$ dor] \\
\hline a. & $/ * \mathrm{n} /$ & & $* !$ & & \\
\hline b. & $/ \mathrm{m} /$ & & & & \\
\hline c. & $/ \mathrm{y} /$ & & $* !$ & $*$ & $*$ \\
\hline
\end{tabular}

C.

\begin{tabular}{|r|c|c|c|c|c|}
\hline \multicolumn{2}{|c|}{ Entrada: $/ \mathrm{y} /$} & $*[$ dor, cor $]$ & FIEL(lug nas) & $*[$ nas, dor $]$ & $*[$ dor $]$ \\
\hline a. & $/ * \mathrm{n} /$ & & $* !$ & & \\
\hline b. & $/ \mathrm{m} /$ & & $* !$ & & \\
\hline c. & $/ \mathrm{y} /$ & & & $*$ & $*$ \\
\hline
\end{tabular}


D.

\begin{tabular}{|r|c|c|c|c|c|c|}
\hline \multicolumn{2}{|c|}{ Entrada: $/ * \mathrm{n} /$} & $*[$ dor, cor $]$ & FIEL(lug nas) & $*[$ nas, dor $]$ & $*[$ dor $]$ & $*[$ lab $]$ \\
\hline a. & $/ * \mathrm{n} /$ & & $*$ & & & \\
\hline b. & $/ \mathrm{m} /$ & & $*$ & & & $* !$ \\
\hline c. & $/ \mathrm{y} /$ & & $*$ & $* !$ & $*$ & \\
\hline d. & $/ * \mathrm{n} /$ & $* !$ & & & & \\
\hline
\end{tabular}

TABLA 17: Generación del inventario /m, *n, y/ (versión final).

guas que tienen este tipo de gramática ubican todos los miembros de la «jerarquía de perceptibilidad nasal» por debajo de Fiel(lugar nasal), las demandas de esa familia de marcación son inconsecuentes en este escenario. Eso fue lo que permitió que el sistema gramatical propuesto en (7) pudiera generar la terna / $\mathrm{m},{ }^{*} \mathrm{n}, \mathrm{y} / \mathrm{mientras}$ se ignoraba por completo la dimensión de la percepción.

Un estado de cosas bastante diferente es el que encontramos en las lenguas que, como el español, completan su terna nasal con / ${ }^{*} \mathrm{n} /$ en vez de /y/. La peculiaridad que tienen tales lenguas es que la «jerarquía de perceptibilidad nasal» asciende de rango, de modo que su presencia se vuelve tangible. Para apreciarlo, comencemos por recordar que, para que la nasal palatal pueda surgir, es necesario que Fiel(lugar nasal) se ubique en una posición relativamente alta con respecto a la «jerarquía articulatoria de lugar»; exactamente, por encima de * [dorsal, coronal], como se estableció anteriormente en (9). Aunque este rango implica que /y/ también debería surgir, eso no sucede en este tipo de gramática porque uno de los miembros de la «jerarquía de perceptibilidad nasal», * [nasal, dorsal], tiene supremacía sobre FIEL (lugar nasal). Es decir que las relaciones cruciales son *[nasal, dorsal] $>$ FIEL (lugar nasal) $>>$ * [dorsal, coronal], como se ilustra en (18). Los demás rangos se desprenden automáticamente de las jerarquías universales.

Las tablas (19A-E) demuestran que la organización gramatical en (18) es capaz de generar el inventario nasal /m, *n, *n/ sin producir ninguna otra CNP. Obsérvese que, si la entrada postulada es /*n/, /m/ $\mathrm{o} /{ }^{*} \mathrm{n} /$, la salida óptima resulta ser el candidato fiel (19Aa, 19Bb y 19Dd), lo cual es posible debido a que las combinaciones del rasgo [nasal] con los rasgos de lugar de esas CNPs tienen un coste perceptivo menor que el umbral protegido por * [nasal, dorsal]. Además de generar $/{ }^{*} \mathrm{n} /$, la otra innovación que vemos en (19) es que, cuando la entrada es $/ *^{*} \mathrm{y} /$, la salida óptima no puede ser el candidato fiel porque la combinación del rasgo [nasal] con el rasgo de lugar de esta CNP tiene un coste per- 


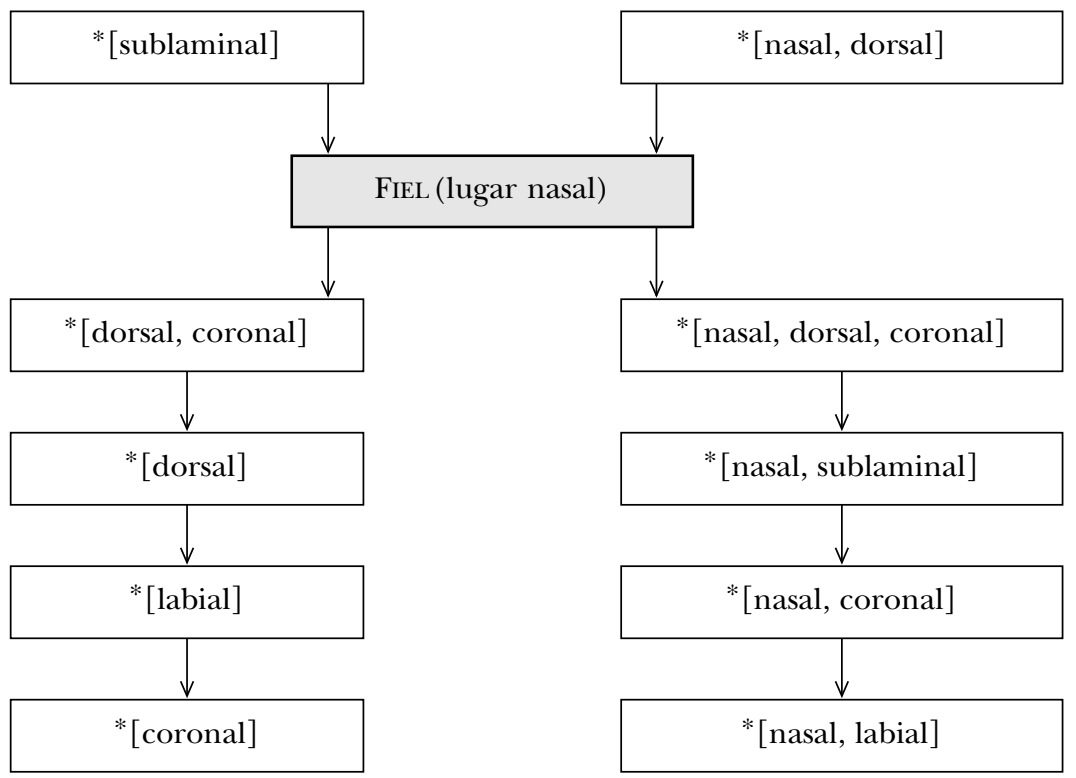

TABLA 18: Gramática de una lengua con el inventario nasal

$$
/ \mathrm{m}, * \mathrm{n},{ }^{*} \mathrm{n} / \text {. }
$$

ceptivo que alcanza el umbral defendido por * [nasal, dorsal]. En este caso, la gramática se inclina por el candidato que opta por volverse infiel por medio de renunciar a [dorsal], el rasgo de lugar perceptivamente más costoso, y adoptar [coronal], el rasgo de lugar articulatoriamente más económico (19Ca).

La tabla (19E) ilustra lo que pasaría si la entrada postulada fuese $/ \mathrm{n} /$, un caso que requiere la presencia de ${ }^{*}$ [sublaminal] para poder evaluar el alófono fiel. Aquí la gramática vuelve a favorecer al candida-

A.

\begin{tabular}{|r|c|c|c|c|c|c|}
\hline \multicolumn{2}{|c|}{ Entrada: $/ * \mathrm{n} /$} & $*[$ nas, dor] & $\begin{array}{c}\text { FIEL } \\
\text { (lug nas })\end{array}$ & $*[$ dor, cor] & $*[$ dor $]$ & $*[$ lab] \\
\hline a. & $/ * \mathrm{n} /$ & & & & & \\
\hline b. & $/ \mathrm{m} /$ & & $* !$ & & & $*$ \\
\hline c. & $/ \mathrm{y} /$ & $* !$ & $*$ & & $*$ & \\
\hline d. & $/ * \mathrm{n} /$ & & $* !$ & $*$ & & \\
\hline
\end{tabular}


B.

\begin{tabular}{|r|c|c|c|c|c|c|}
\hline \multicolumn{2}{|c|}{ Entrada: $/ \mathrm{m} /$} & $*[$ nas, dor $]$ & $\begin{array}{c}\text { FIEL } \\
(\text { lug nas })\end{array}$ & $*[$ dor, cor $]$ & $*[$ dor $]$ & $*[$ lab $]$ \\
\hline a. & $/ * \mathrm{n} /$ & & $* !$ & & & \\
\hline $\mathrm{b}$. & $/ \mathrm{m} /$ & & & & & $*$ \\
\hline $\mathrm{c}$. & $/ \mathrm{y} /$ & $* !$ & $*$ & & $*$ & \\
\hline $\mathrm{d}$. & $/ * \mathrm{n} /$ & & $* !$ & $*$ & & \\
\hline
\end{tabular}

C.

\begin{tabular}{|c|c|c|c|c|c|c|}
\hline \multicolumn{2}{|c|}{ Entrada: /n/ } & $*[$ nas, dor $]$ & $\begin{array}{c}\text { FIEL } \\
\text { (lug nas) }\end{array}$ & $*[$ dor, cor $]$ & $*[$ dor $]$ & $*[\mathrm{lab}]$ \\
\hline a. & $/ * \mathrm{n} /$ & & $*$ & & & \\
\hline b. & $/ \mathrm{m} /$ & & $*$ & & & $* !$ \\
\hline c. & $/ \mathrm{y} /$ & $* !$ & & & * & \\
\hline d. & $/ * \mathrm{n} /$ & & $* !$ & * & & \\
\hline
\end{tabular}

D.

\begin{tabular}{|r|c|c|c|c|c|c|}
\hline \multicolumn{2}{|c|}{ Entrada: $/ * \mathrm{n} /$} & $*$ [nas, dor $]$ & $\begin{array}{c}\text { FiEL } \\
\text { (lug nas })\end{array}$ & $*[$ dor, cor $]$ & $*[$ dor $]$ & $*[$ lab $]$ \\
\hline a. & $/ * \mathrm{n} /$ & & $* !$ & & & \\
\hline $\mathrm{b}$. & $/ \mathrm{m} /$ & & $* !$ & & & $*$ \\
\hline $\mathrm{c}$. & $/ \mathrm{y} /$ & $* !$ & & & $*$ & \\
\hline $\mathrm{d}$. & $/ * \mathrm{n} /$ & & & $*$ & & \\
\hline
\end{tabular}

E.

\begin{tabular}{|r|c|c|c|c|c|c|c|}
\hline \multicolumn{2}{|c|}{ Entrada: $/ \mathrm{n} /$} & $*[$ nas, dor $]$ & $*[$ sblm $]$ & $\begin{array}{c}\text { FIEL } \\
\text { (lug nas) }\end{array}$ & $*[$ dor, cor $]$ & $*[$ dor $]$ & $*[$ lab $]$ \\
\hline a. & $/ * \mathrm{n} /$ & & & $*$ & & & \\
\hline b. & $/ \mathrm{m} /$ & & & $*$ & & & $* !$ \\
\hline c. & $/ \mathrm{n} /$ & $* !$ & & $*$ & & $*$ & \\
\hline d. & $/ * \mathrm{n} /$ & & & $*$ & $* !$ & & \\
\hline e. & $/ \mathrm{n} /$ & & $* !$ & & & & \\
\hline
\end{tabular}

TABLA 19: Generación del inventario /m, *n, *n/. 
to que opta por volverse infiel por medio de adoptar el rasgo de lugar menos marcado articulatoriamente (19Ea). La diferencia con respecto a (19C) es que, en este caso, la causa de la infidelidad no es que / n/ tenga un coste perceptivo superior al umbral permitido, sino que su coste articulatorio sobrepasa el umbral que la gramática puede tolerar cuando FIEL (lugar nasal) está por debajo de *[sublaminal]. Es esa restricción la que descarta al candidato fiel (19Ee).

Las lenguas que, como el telugu, completan la terna nasal con / / en vez de /y/ requieren que la gramática autorice no solo un ascenso mayor en el rango de FiEL (lugar nasal) con respecto a la «jerarquía articulatoria de lugar», sino también un descenso mayor con respecto a la «jerarquía de perceptibilidad nasal». Compárese el diagrama en (20) con los de (18) y (16).

El diagrama en (20) muestra que FiEL(lugar nasal) pasa a dominar a *[sublaminal], de modo que ahora el lugar retroflejo ya no queda vetado. Además, puesto que ${ }^{*}$ [nasal, dorsal, coronal] pasa a dominar a

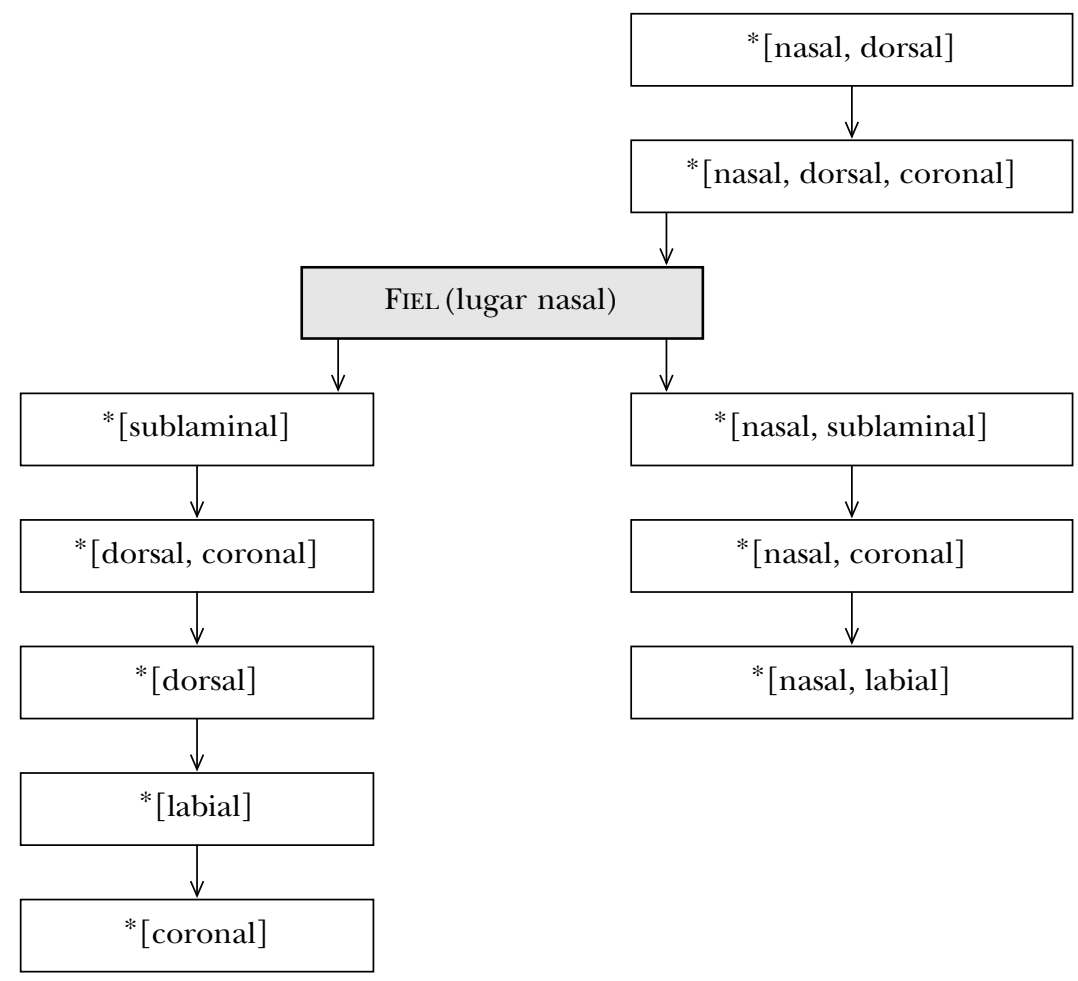

TABLA 20: Gramática de una lengua con el inventario nasal /m, *n, n/. 
FIEL(lugar nasal), ni / $\mathrm{n} / \mathrm{ni} /{ }^{*} \mathrm{n} /$ son opciones perceptivamente viables. La consecuencia de esta reducción en el umbral de perceptibilidad es que /n/ emerge como la CNP más posterior que la gramática puede derivar. En breve, las relaciones * [nasal, dorsal, coronal] $>$ FIEL(lugar nasal) $\gg *$ [sublaminal $]$ son las cruciales para este tipo de lengua.

Las tablas (21A-D) demuestran la generación del inventario $/ \mathrm{m},{ }^{*} \mathrm{n}$, n/ a través de este análisis. Cuando la entrada consiste en una CNP cuya oclusión oral es bastante anterior por ser ejecutada por la corona, los labios o la sublámina $-/{ }^{*} \mathrm{n} /, / \mathrm{m} / \mathrm{y} / \mathrm{n} /-$, el candidato fiel es el que resulta seleccionado (21Aa, 21Bb, 21Ee). En contraste, cuando la entrada consiste en una CNP cuya oclusión oral es bastante posterior por ser ejecutada por el dorso $-/ \mathrm{y} / \mathrm{y} / * \mathrm{n} /-$, el candidato que opta por volverse infiel renunciando al rasgo [dorsal] y adoptando el rasgo [coronal] es el elegido (21Ca, 21Da). El punto más importante es que, como todos los miembros de la «jerarquía articulatoria de lugar» están ubicados por debajo de FiEL(lugar nasal), la «jerarquía de perceptibilidad nasal» es la que en último lugar selecciona los integrantes del inventario nasal de tales lenguas.

A.

\begin{tabular}{|r|c|c|c|c|c|c|c|}
\hline \multicolumn{2}{|c|}{ Entrada: $/ * \mathrm{n} /$} & $*[$ nas, dor, cor] & $\begin{array}{c}\text { FIEL } \\
\text { (lug nas) }\end{array}$ & $*[$ sblm $]$ & $*[$ dor, cor $]$ & $*[$ dor $]$ & $*[$ lab $]$ \\
\hline a. & $/ * \mathrm{n} /$ & & & & & & \\
\hline $\mathrm{b}$. & $/ \mathrm{m} /$ & & $* !$ & & & & $*$ \\
\hline $\mathrm{c}$. & $/ \mathrm{n} /$ & $* !$ & $*$ & & & $*$ & \\
\hline $\mathrm{d}$. & $/ * \mathrm{n} /$ & $* !$ & $*$ & & $*$ & & \\
\hline $\mathrm{e}$. & $/ \mathrm{n} /$ & & $* !$ & $*$ & & & \\
\hline
\end{tabular}

B.

\begin{tabular}{|r|c|c|c|c|c|c|c|}
\hline \multicolumn{2}{|c|}{ Entrada: $/ * \mathrm{~m} /$} & $*[$ nas, dor, cor $]$ & $\begin{array}{c}\text { FIEL } \\
\text { (lug nas) }\end{array}$ & $*[$ sblm $]$ & $*[$ dor, cor $]$ & $*[$ dor $]$ & $*[$ lab $]$ \\
\hline a. & $/ * \mathrm{n} /$ & & $* !$ & & & & \\
\hline b. & $/ \mathrm{m} /$ & & & & & & $*$ \\
\hline $\mathrm{c}$. & $/ \mathrm{n} /$ & $* !$ & $*$ & & & $*$ & \\
\hline $\mathrm{d}$. & $/ * \mathrm{n} /$ & $* !$ & $*$ & & $*$ & & \\
\hline $\mathrm{e}$. & $/ \mathrm{n} /$ & & $* !$ & $*$ & & & \\
\hline
\end{tabular}


C.

\begin{tabular}{|r|c|c|c|c|c|c|c|}
\hline \multicolumn{2}{|c|}{ Entrada: $/ * \mathrm{n} /$} & $*[$ nas, dor, cor $]$ & $\begin{array}{c}\text { FIEL } \\
(\text { lug nas })\end{array}$ & $*[$ sblm $]$ & $*[$ dor, cor $]$ & $*[$ dor $]$ & $*[$ lab] \\
\hline a. & $/ * \mathrm{n} /$ & & $*$ & & & & \\
\hline $\mathrm{b}$. & $/ \mathrm{m} /$ & $* !$ & $*$ & & & & $* !$ \\
\hline $\mathrm{c}$. & $/ \mathrm{y} /$ & $* !$ & $*$ & & $*$ & & \\
\hline $\mathrm{d}$. & $/ * \mathrm{n} /$ & & $*$ & $* !$ & & & \\
\hline $\mathrm{e}$. & $/ \mathrm{n} /$ & & & & & & \\
\hline
\end{tabular}

D.

\begin{tabular}{|r|c|c|c|c|c|c|c|}
\hline \multicolumn{2}{|c|}{ Entrada: $/ * \mathrm{n} /$} & $*[$ nas, dor, cor $]$ & $\begin{array}{c}\text { FIEL } \\
(\text { lug nas })\end{array}$ & $*[\mathrm{sblm}]$ & $*[$ dor, cor $]$ & $*[$ dor $]$ & $*[$ lab $]$ \\
\hline a. & $/ * \mathrm{n} /$ & & $*$ & & & & \\
\hline $\mathrm{b}$. & $/ \mathrm{m} /$ & $* !$ & $*$ & & & & $* !$ \\
\hline $\mathrm{c}$. & $/ \mathrm{y} /$ & $* !$ & & & $*$ & & \\
\hline d. & $/ * \mathrm{n} /$ & & $*$ & $* !$ & & & \\
\hline e. & $/ \mathrm{n} /$ & & & & & & \\
\hline
\end{tabular}

E.

\begin{tabular}{|r|c|c|c|c|c|c|c|}
\hline \multicolumn{2}{|c|}{ Entrada: $/ * \mathrm{n} /$} & $*$ [nas, dor, cor $]$ & $\begin{array}{c}\text { FIEL } \\
(\text { lug nas })\end{array}$ & $*[\mathrm{sblm}]$ & $*[$ dor, cor $]$ & $*[$ dor $]$ & $*[$ lab] \\
\hline $\mathrm{a}$. & $/ * \mathrm{n} /$ & & $* !$ & & & & \\
\hline $\mathrm{b}$. & $/ \mathrm{m} /$ & & $* !$ & & & & $*$ \\
\hline $\mathrm{c}$. & $/ \mathrm{n} /$ & $* !$ & $*$ & & & $*$ & \\
\hline $\mathrm{d}$. & $/ * \mathrm{n} /$ & $* !$ & $*$ & & $*$ & & \\
\hline $\mathrm{e}$. & $/ \mathrm{n} /$ & & & $*$ & & & \\
\hline
\end{tabular}

TABLA 21: Generación del inventario /m, *n, *n/.

Para cerrar esta sección, vale la pena acentuar que el análisis que acabamos de presentar respeta estrictamente las relaciones de dominio entre los miembros de las jerarquías universales propuestas en (5) y (15). En ningún caso se ha recurrido a manipular esas jerarquías para obtener los resultados deseados. Hemos visto que las diferencias en cuanto 
a la cantidad y la cualidad de las unidades que componen los inventarios nasales proceden de los diferentes rangos que los miembros de las jerarquías universales de marcación pueden tener con respecto a la restricción Fiel (lugar nasal), lo cual no es más que una instancia del perpetuo conflicto gramatical que existe entre los principios lingüísticos de MARCACIÓN y FIDELIDAD.

\section{LA PRIMACÍA DE $/ * \mathrm{n} / \mathrm{y} / \mathrm{m} /$}

En las secciones 1 y 3 mencionamos que $/{ }^{*} \mathrm{n} / \mathrm{y} / \mathrm{m} /$ son las dos CNPs más comunes que existen. Ocurren en las lenguas de la UPSID con una frecuencia de $98.67 \%$ y $94.24 \%$, respectivamente (consúltese la sección 6). La explicación para la superabundancia de estas dos CNPs se deriva de las jerarquías en (5) y (15). La ubicación de la restricción * [coronal] en el extremo inferior de la «jerarquía articulatoria de lugar» garantiza que ninguna otra CNP tendrá un menor coste articulatorio para la gramática que $/{ }^{*} \mathrm{n} /$. Del mismo modo, al estar ubicada en el extremo inferior de la «jerarquía de perceptibilidad nasal», la restricción * [nasal, labial] asegura que el coste perceptivo de /m/ será inferior al de cualquier otra CNP. Es decir que, bajo el supuesto de que paralela a la dimensión de marcación articulatoria existe una dimensión de marcación perceptiva, la expectativa es que no haya una sola, sino dos CNPs que se comporten como si gozaran de un estatus especial.

Además de ser extremadamente abundantes, otro hecho que apunta al estatus no marcado tanto de $/{ }^{*} \mathrm{n} /$ como de $/ \mathrm{m} /$ es que no todas las lenguas que construyen su inventario nasal con una sola unidad eligen la misma CNP. Entre el pequeño grupo de lenguas de la UPSID que emplean una sola CNP (9, que equivale al $2 \%$ ), hay 7 que eligen / ${ }^{*} \mathrm{n} /$ y 2 que eligen $/ \mathrm{m} /{ }^{11}$. El punto de interés es que, si no contásemos más que con la «jerarquía articulatoria de lugar», los inventarios nasales en los que / $\mathrm{m}$ / figura como el único integrante serían un enigma porque no tendríamos ningún argumento para justificar su preeminencia sobre $/{ }^{*} \mathrm{n} /$. Con la adopción de la «jerarquía de perceptibilidad nasal», en cambio, los inventarios nasales compuestos exclusivamente por /m/ son un resultado esperado. Para verificarlo, compárense (22) y (23).

En (22), FiEL(lugar nasal) fuerza la selección de $/{ }^{*} \mathrm{n} /$ porque domi-

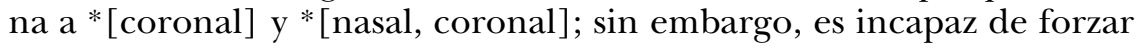
la selección de /m/ porque *[labial] la sobrepasa en rango. El resulta-

\footnotetext{
${ }_{11}$ Mixtec es un caso especial porque se ha reportado que su única CNP es / $\mathrm{n} /$. Maddieson 1984, p. 62 aclara, sin embargo, que es probable que la consonante en cuestión no sea un segmento subyacente sino derivado, lo cual significaría que Mixtec es en realidad una lengua que carece completamente de CNPs. Es así como la hemos clasificado aquí.
} 


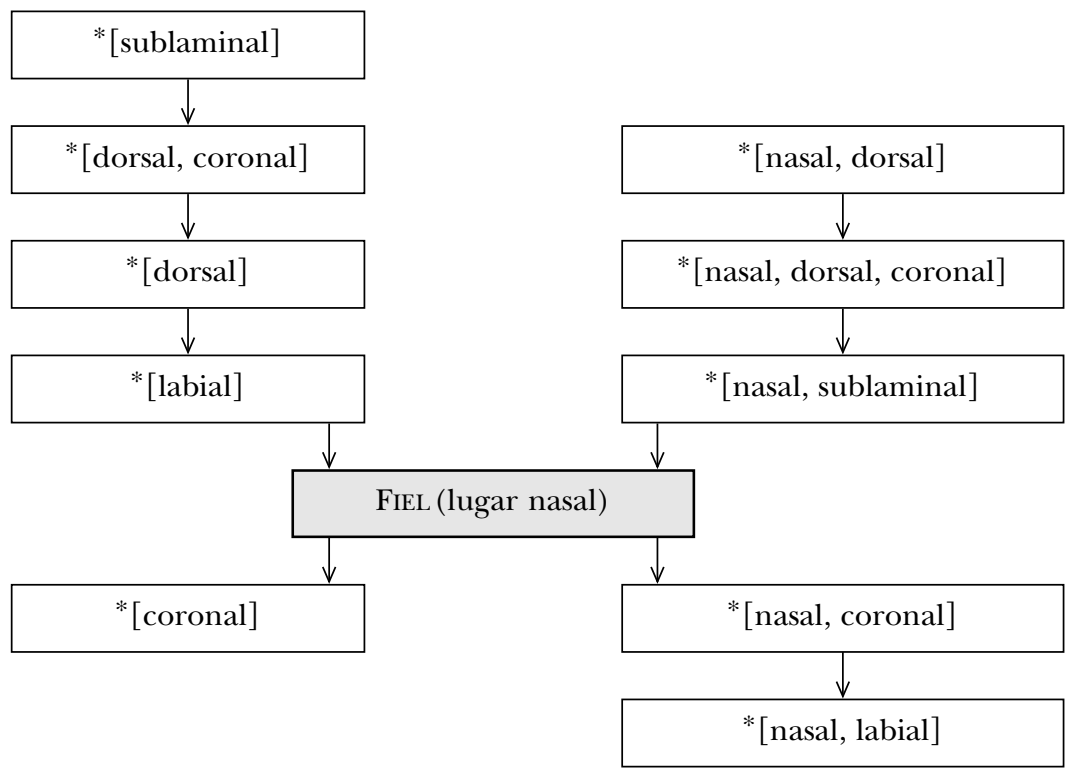

TABLA 22: Gramática de una lengua con el inventario nasal /*n/.

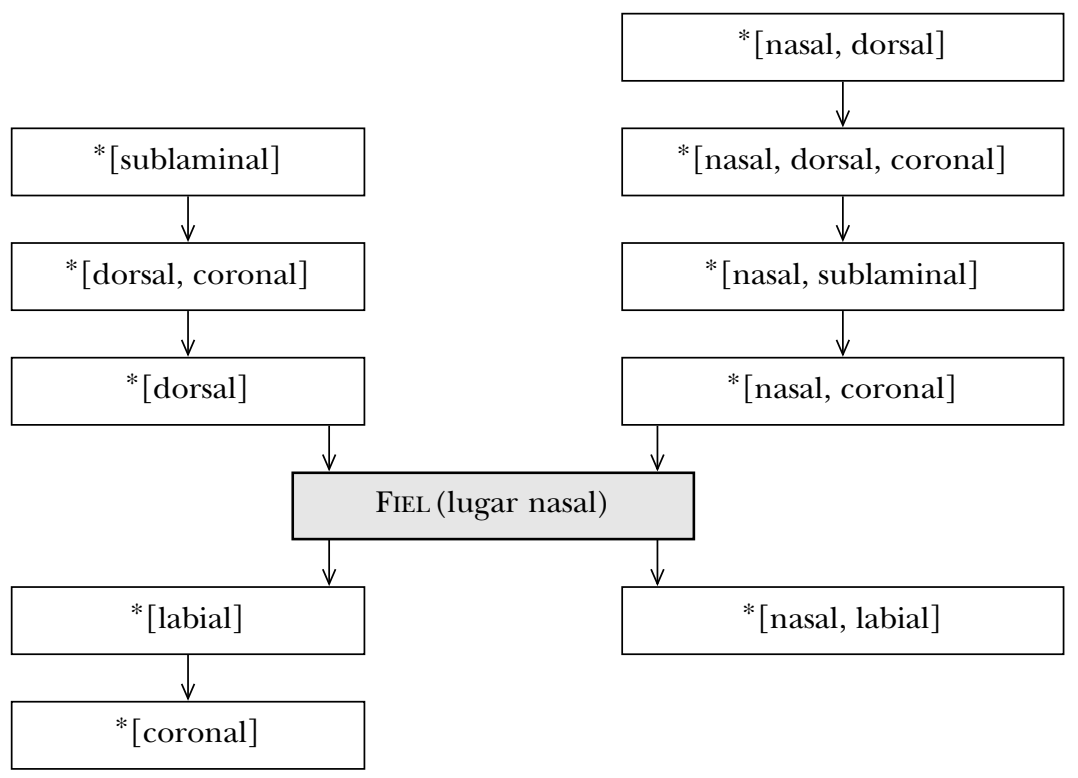

TABLA 23: Gramática de una lengua con el inventario nasal /*m/. 
do en (23) es justamente el reverso. Fiel(lugar nasal) no puede forzar la selección de /*n/ porque está por debajo de *[nasal, coronal], pero logra forzar la selección de /m/ porque está por encima de *[labial] $y^{*}$ [nasal, labial]. La importancia teórica de estos resultados es que no hubo que apelar a manipulaciones arbitrarias de la «jerarquía articulatoria de lugar»; es decir, que la presente propuesta obvia la necesidad de tener que estipular que, para algunas lenguas, [coronal] es el rasgo de lugar articulatoriamente menos marcado; mientras que, para otras lenguas, [labial] es el rasgo de lugar que tiene ese privilegio. Nuestro análisis revela que la aparente contradicción no es más que una ilusión creada por el hecho de que existen múltiples dimensiones de marcación relevantes para las consonantes nasales; mínimamente, una dimensión articulatoria y otra perceptiva.

Las lenguas cuyo inventario nasal consta del par $/ \mathrm{m},{ }^{*} \mathrm{n} /$ corresponden al estado ilustrado en (24), en donde la restricción FIEL (lugar nasal) domina a las dos restricciones más bajas de cada una de las jerarquías universales de marcación. El efecto que esto tiene es que, a diferencia de lo que ocurre en (22) y (23), las dos familias de marcación no se contradicen sino que concuerdan en las CNPs que eligen. Debido a que tanto la «jerarquía articulatoria de lugar» como «la jerarquía de perceptibilidad nasal» reservan sus dos niveles más bajos para las restricciones que prohíben los rasgos [coronal] y [labial], el desacuerdo que normalmente existe entre estas jerarquías desaparece cuando su rango con respecto a FIEL (lugar nasal) les permite seleccionar dos CNPs.

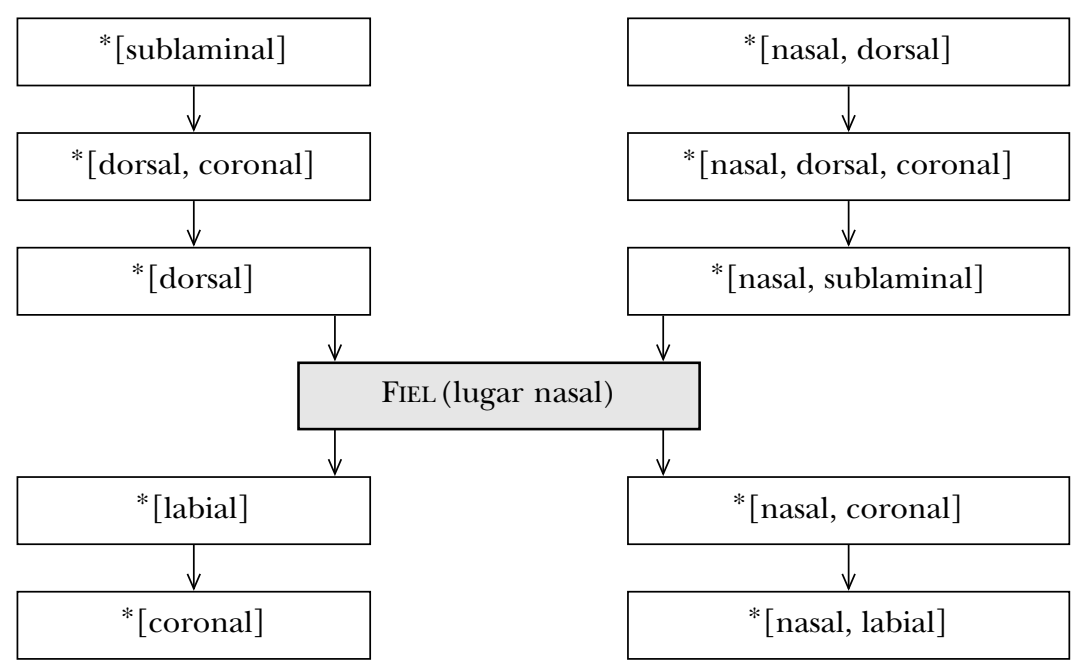

TABLA 24: Gramática de una lengua con el inventario nasal /m, *n/. 
En otras palabras, desde el punto de vista articulatorio como desde el punto de vista perceptivo, /m/ y / $\mathrm{n} /$ son las dos CNPs óptimas.

Ya vimos en la sección 4 que, cuando Fiel(lugar nasal) asciende a una posición más alta con respecto a las dos familias de marcación, el desacuerdo vuelve a presentarse. Según la medida en que FiEL (lugar nasal) sea promovida, la tercera CNP elegida puede ser $/ \mathrm{y} /, / \mathrm{n} / \mathrm{o} / \mathrm{n} /$, como vimos en (16), (18) y (20).

Prosiguiendo con el movimiento ascendente de Fiel (lugar nasal), se llega a los inventarios nasales compuestos por cuatro y cinco CNPs (25 y 26), donde el dominio de FiEL(lugar nasal) es tal que obliga a las CNPs menos marcadas a coexistir con las más marcadas. Por motivos de espacio, no incluimos aquí las tablas que ilustran la selección de los inventarios $/ \mathrm{m},{ }^{*} \mathrm{n}, \mathrm{\eta},{ }^{*} \mathrm{n} / \mathrm{y} / \mathrm{m},{ }^{*} \mathrm{n}, \mathrm{n},{ }^{*} \mathrm{n}, \mathrm{n} /$, pero es evidente que si * [sublaminal] es la única restricción de (5) y (15) que no sucumbe ante FiEL(lugar nasal), / / será la única CNP de la entrada que se verá excluida (25). Si, por otro lado, todas las restricciones de (5) y (15) sucumben ante FiEL(lugar nasal), ninguna de las CNPs incluidas en la entrada quedará excluida (26).

Existe también la posibilidad de que FIEL(lugar nasal) quede dominada por todos los miembros de ambas jerarquías de marcación (27),

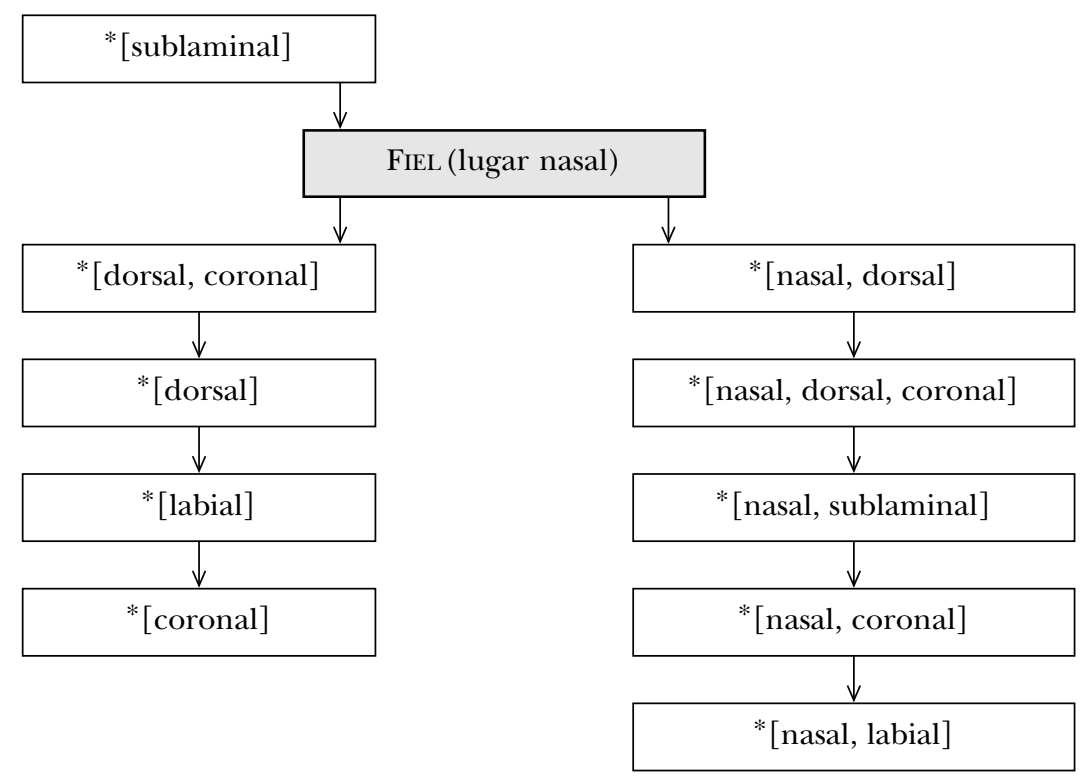

TABLA 25: Gramática de una lengua con el inventario nasal / $\mathrm{m}$, *n, $\mathrm{y}$, *n/ (versión final). 


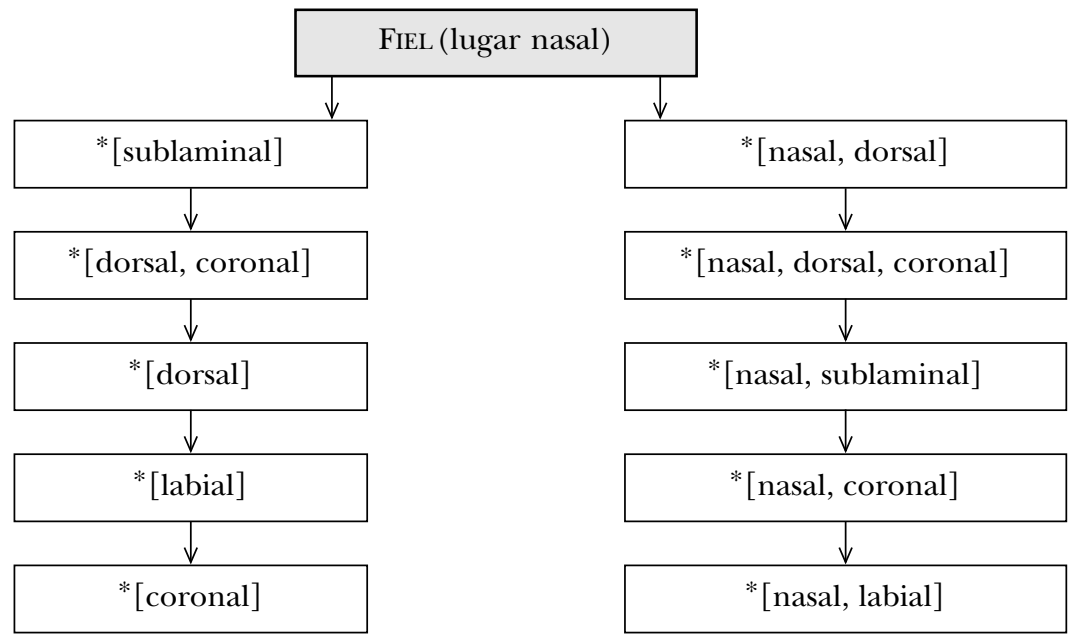

TABLA 26: Gramática de una lengua con el inventario nasal /m, *n, y, *n, n/ (versión final).

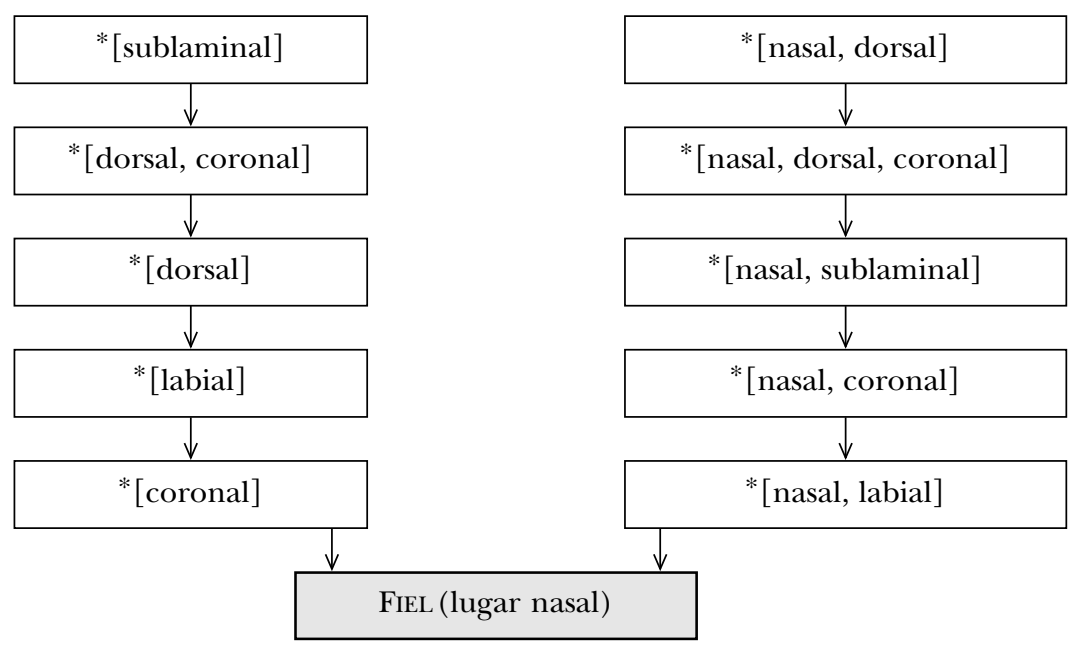

TABLA 27: Gramática de una lengua carente de CNPs.

en cuyo caso ni las CNPs con los rasgos de lugar menos marcados pueden surgir. Tal gramática corresponde, pues, al grupo minoritario de lenguas que no emplean ninguna CNP $(3.77 \%)$. 
Como una anotación final, cabe resaltar que el análisis propuesto aquí no cae en el error de sobre-generar; o sea que no produce inventarios nasales inexistentes. Por ser universales, las jerarquías de marcación en (5) y (15) descartan categóricamente la posibilidad de que una lengua humana construya su inventario nasal seleccionando exclusivamente CNPs marcadas (p. ej. la terna /n, *n, $\mathrm{n} /$, el par $/ \eta$, *n/, o la unidad / $/$ ). Bajo los supuestos que hemos adoptado, las CNPs con los rasgos de lugar más marcados solo podrán surgir en inventarios nasales que también incluyan las CNPs con los rasgos de lugar menos marcados. Dado que ninguna de las 451 lenguas de la UPSID contradice esta predicción, la universalidad de las jerarquías propuestas en (5) y (15) se ve fuertemente apoyada.

\section{Conclusión}

Este trabajo ha investigado la composición del inventario fonémico nasal del español usando como marco de comparación los inventarios de las lenguas de la UPSID. Se encontró que el inventario /m, *n, *n/ concuerda con una tendencia translingüística predominante -la inclusión de $/ \mathrm{m} / \mathrm{y} /{ }^{*} \mathrm{n} /-$ pero discrepa de otra tendencia translingüística predominante -la elección de / $\mathrm{y} /$ para completar la terna nasal-. De acuerdo con estos hallazgos, las preguntas de investigación que se plantearon fueron las siguientes.

1. ¿Cuáles son los principios universales responsables de que el español, al igual que la mayoría de las lenguas del mundo, incluya $/ \mathrm{m} / \mathrm{y} /{ }^{*} \mathrm{n} /$ en su inventario nasal?

2. ¿Qué es lo que hace que las lenguas representadas por el español se alejen de la tendencia predominante a completar la terna nasal con / $\mathrm{y} / \mathrm{y}$ opten por incluir /* $\mathrm{n} /$ en su lugar?

3. ¿Cuál es la razón de que las lenguas representadas por el telugu

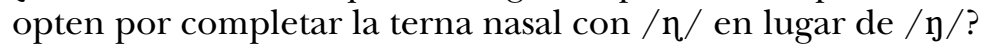

4. ¿Es acaso posible que el factor causante de tales desviaciones sea el mismo?

Las respuestas a estos interrogantes están relacionadas con la marcación de los rasgos distintivos de lugar: [labial] para /m/, [coronal] para $/ * \mathrm{n} /$, [dorsal] para / $\mathrm{n} /$, , [dorsal, coronal] para /*n/ y [sublaminal] para $/ n /$. La base argumental de nuestro análisis es el supuesto de que, además de la dimensión de marcación articulatoria -formalizada como la jerarquía en (5)-, existe una dimensión de marcación perceptiva -formalizada como la jerarquía en (15)-. Las discrepancias en el resultado de someter las consonantes nasales a evaluación por parte de 
estas dos familias de principios son inevitables debido a que el orden que la «jerarquía articulatoria de lugar» les asigna a las restricciones que prohíben los rasgos de lugar de acuerdo con criterios articulatorios no coincide con el orden que la «jerarquía de perceptibilidad nasal» les asigna a las restricciones que prohíben los rasgos de lugar de acuerdo con criterios perceptivos. Una de las consecuencias que emanan de este desfase entre la producción y la percepción de consonantes nasales es que, mientras que [coronal] es el rasgo de lugar menos marcado articulatoriamente, [labial] es el rasgo de lugar menos marcado perceptivamente.

Se demostró que el número de consonantes nasales y los rasgos de lugar que estas puedan tener dependen del rango que cada gramática le asigne a la restricción FIEL(lugar nasal) con respecto a los integrantes de las dos jerarquías universales de marcación. Según este enfoque, la dominación absoluta de Fiel(lugar nasal) por parte de ambas jerarquías de marcación acarrea la ausencia de CNPs (27), mientras que la promoción gradual de esa restricción de fidelidad conduce a inventarios nasales gradualmente más poblados.

Cuando FiEL(lugar nasal) sobrepasa solamente los niveles más bajos de cada jerarquía de marcación, su habilidad para proteger los rasgos de lugar de las consonantes nasales es bastante limitada. Además, como los rasgos de lugar prohibidos en el nivel más bajo de cada jerarquía de marcación son diferentes, se presenta la posibilidad de que la unidad seleccionada sea $/{ }^{*} \mathrm{n} / \mathrm{o} / \mathrm{m} /$ dependiendo de si la gramática opta por reducir el coste articulatorio a expensas del coste perceptivo o viceversa (22 y 23). La inclusión de dos CNPs se obtiene una vez que FiEL (lugar nasal) se ubica por encima de los dos niveles más bajos de cada jerarquía de marcación (24), en cuyo caso las unidades seleccionadas son $/{ }^{*} \mathrm{n} / \mathrm{y} / \mathrm{m} /$ debido a que sus rasgos de lugar son los dos valores más económicos, tanto en la dimensión articulatoria como en la dimensión perceptiva.

La terna nasal más común, /m, *n, y/, responde a una promoción aun más alta de Fiel (lugar nasal) y se caracteriza, además, por una inclinación a salvaguardar la dimensión de marcación articulatoria a expensas de la dimensión de marcación perceptiva (16). El factor que conduce a la selección de / $\mathrm{y} /$ como el tercer integrante de la terna nasal es que FiEL(lugar nasal) se impone sobre todos los miembros de la «jerarquía de perceptibilidad nasal», de modo que aunque /y/ es la CNP perceptivamente más costosa, tal derroche es permisible. Una situación alternativa es la que encontramos en las lenguas como el espanol (18), donde cuatro restricciones de cada jerarquía de marcación se someten al dominio de FiEL (lugar nasal). Esto asegura que ningún miembro de la terna resultante, /m, *n, *n/, alcanzará el máximo nivel en ninguna de las dos dimensiones de marcación. A partir de esto es posible inferir que la selección de $/ * \mathrm{n} /$ sobre / $\mathrm{y} /$ es una manera de 
equilibrar el coste articulatorio y perceptivo acarreado por la inclusión de una tercera unidad. En las lenguas representadas por el telugu, por otro lado, la gramática opta por adoptar la tercera alternativa lógica; esto es, favorecer la dimensión de marcación perceptiva a expensas de la dimensión de marcación articulatoria (20). El efecto que esto tiene es forzar la inclusión de la consonante nasal con el rasgo de lugar articulatoriamente más marcado, [sublaminal], de modo que la terna que se obtiene es /m, *n, n/. Mayores ascensos de FIEL(lugar nasal) con respecto a ambas jerarquías de marcación resultan, obviamente, en inventarios nasales más copiosos y marcados, como son el cuarteto /m, *n, $\mathrm{n},{ }^{*} \mathrm{n} / \mathrm{y}$ el quinteto /m, *n, n, *n, n/ (25 y 26$)$.

La generación de esta amplia gama de inventarios nasales no significa, sin embargo, que el poder de las restricciones postuladas sea ilimitado. Por alto que sea el rango que se le otorgue a la restricción Fiel (lugar nasal), el carácter universal con el que las jerarquías de marcación han sido consideradas descarta categóricamente la posibilidad de generar inventarios nasales constituidos exclusivamente por unidades marcadas (p. ej. / $\eta, * n, \eta \mathrm{y} /$ ). Solo en los inventarios que incluyen las CNPs menos marcadas pueden las CNPs más marcadas surgir también.

\section{REFERENCIAS BIBLIOGRÁFICAS}

Albalá, M. J. (1992): «Análisis y síntesis de las consonantes nasales», Revista de filología española 72, pp. 37-55.

DE LACY, P. (2002): The formal expression of scales. Ph.D. dissertation, University of Massachusetts, Amherst.

Ferguson, C. (1963): «Assumptions about nasals: A sample study in phonological universals», en Greenberg, J. (ed.), Universals of language. M.I.T. Press, Cambridge, pp. 53-60.

FujIMORA, O. (1962): «Analysis of nasal consonants», Journal of the Acoustical Society of America 34, pp. 1865-1875.

Johnson, K., DiCanio, C. y MaCKenzie, L. (2007): «The acoustic and visual phonetic basis of place of articulation in excrescent nasals», UC Berkeley Phonology Lab Annual Report, pp. 529-561.

House, A. (1957): «Analog studies of nasal consonants», Journal of Speech and Hearing Disorders 22, pp. 190-204.

JuN, J. (1995): «Place assimilation as the result of conflicting perceptual and articulatory constraints», West Coast Conference on Formal Linguistics 14, pp. 221-237.

Kurowski, K. M. y Blumstein, S. E. (1984): «Perceptual integration of the murmur and formant transitions for place of articulation in nasal consonants», Journal of the Acoustical Society of America 76, pp. 383-390.

- (1987): «Acoustic properties of place of articulation in nasal consonants», Journal of the Acoustical Society of America 81, pp. 1917-1927. 
- (1993): «Acoustic properties for the perception of nasal consonants», en Huffman, M. y Krakow, R. (eds.), Phonetics and Phonology 5, Nasals, Nasalization, and the Velum, San Diego, CA, Academic Press, Inc., pp. 225-249.

Maddieson, I. (1984): Patterns of sounds, Cambridge University Press, Cambridge.

MALÉCOT, A. (1956): «Acoustic cues for nasal consonants: An experimental study involving tape-splicing technique», Language 32, pp. 271-284.

Massone, M. I. (1988): «Estudio acústico y perceptivo de las consonantes nasales y líquidas del español», Estudios de Fonética Experimental 3, pp. 13-34.

NORD, L. (1976): «Perceptual experiments with nasals. Stockholm, Royal Institute of Technology, Speech Transmission Laboratory», Quarterly Progress Status Report, pp. 2-3.

OHALA, J. (1975): «Phonetic explanations for nasal sound patterns», en Ferguson, C., Hyman, L. y Ohala, J. (eds.), Nasalfest, Stanford University, Stanford, pp. 289-316.

- y Ohala, M. (1993): «The phonetics of nasal phonology: Theorems and data», en Huffman, M. y Krakow, R. (eds.), Phonetics and Phonology 5, Nasals, Nasalization, and the Velum, San Diego, CA, Academic Press, Inc., pp. 225-249.

Prince, A. y SMOlensky, P. (1993): Optimality theory: constraint interaction in generative grammar, Ms., Technical Report \# 2 of the Rutgers Center for Cognitive Science, Rutgers University. (Publicado también en 2002, con el mismo título, en Rutgers Optimality Archive, 537-0802, 262 pp.)

RECASSENS, D. (1983): «Place cues for nasal consonants with special reference to Catalan», Journal of the Acoustical Society of America 73, pp. 1346-1353. 
